Article

\title{
Scope Out Multiband Gravitational-Wave Observations of GW190521-Like Binary Black Holes with Space Gravitational Wave Antenna B-DECIGO
}

\author{
Hiroyuki Nakano ${ }^{1, *(\mathbb{D})}$, Ryuichi Fujita ${ }^{2,3}\left(\mathbb{D}\right.$, Soichiro Isoyama ${ }^{4}\left(\mathbb{D}\right.$ and Norichika Sago ${ }^{5,6}(\mathbb{C}$ \\ 1 Faculty of Law, Ryukoku University, Kyoto 612-8577, Japan \\ 2 Institute of Liberal Arts, Otemon Gakuin University, Osaka567-8502, Japan; \\ ryuichi.fujita@yukawa.kyoto-u.ac.jp \\ 3 Center for Gravitational Physics, Yukawa Institute for Theoretical Physics, Kyoto University, \\ Kyoto 606-8502, Japan \\ 4 School of Mathematics, University of Southampton, Southampton SO17 1BJ, UK; \\ isoyama@yukawa.kyoto-u.ac.jp \\ 5 Department of Physics, Kyoto University, Kyoto 606-8502, Japan; sago@tap.scphys.kyoto-u.ac.jp \\ 6 Advanced Mathematical Institute, Osaka City University, Osaka 558-8585, Japan \\ * Correspondence: hinakano@law.ryukoku.ac.jp
}

check for updates

Citation: Nakano, H.; Fujita, R.; Isoyama, S.; Sago, N. Scope Out Multiband Gravitational-Wave Observations of GW190521-Like Binary Black Holes with Space Gravitational Wave Antenna B-DECIGO. Universe 2021, 7, 53. https://doi.org/10.3390/universe 7030053

Academic Editor: Ofek Birnholtz

Received: 16 January 2021

Accepted: 23 February 2021

Published: 3 March 2021

Publisher's Note: MDPI stays neutral with regard to jurisdictional claims in published maps and institutional affiliations.

Copyright: (c) 2021 by the authors. Licensee MDPI, Basel, Switzerland. This article is an open access article distributed under the terms and conditions of the Creative Commons Attribution (CC BY) license (https:// creativecommons.org/licenses/by/ $4.0 /)$.

\begin{abstract}
The gravitational wave event, GW190521, is the most massive binary black hole merger observed by ground-based gravitational wave observatories LIGO/Virgo to date. While the observed gravitational wave signal is mainly in the merger and ringdown phases, the inspiral gravitational wave signal of the GW190521-like binary will be more visible to space-based detectors in the lowfrequency band. In addition, the ringdown gravitational wave signal will be louder in the next generation (3G) of ground-based detectors in the high-frequency band, displaying the great potential of multiband gravitational wave observations. In this paper, we explore the scientific potential of multiband observations of GW190521-like binaries with a milli-Hz gravitational wave observatory: LISA; a deci-Hz observatory: B-DECIGO; and (next generation of) hecto-Hz observatories: aLIGO and ET. In the case of quasicircular evolution, the triple-band observations of LISA, B-DECIGO, and ET will provide parameter estimation errors of the masses and spin amplitudes of component black holes at the level of order of $1-10 \%$. This would allow consistency tests of general relativity in the strong field at an unparalleled precision, particularly with the "B-DECIGO + ET" observation. In the case of eccentric evolution, the multiband signal-to-noise ratio found in "B-DECIGO + ET" observation would be larger than 100 for a five-year observation prior to coalescence, even with high final eccentricities.
\end{abstract}

Keywords: gravitational waves; binary black holes; quasinormal modes; general relativity

\section{Introduction}

Among gravitational wave (GW) events detected by LIGO and Virgo during O1, O2, and O3a runs [1,2], a binary black hole (BBH) merger, GW190521 [3,4], is one of the most striking discoveries. GW190521 is the heaviest BBH merger ever observed, producing a remnant black hole $(\mathrm{BH})$ with a mass of $142_{-16}^{+28} M_{\odot}$ that can be interpreted as an intermediate mass BH; the source parameters of GW190521 (and our notations) are summarized in Table 1. This measurement triggers the intense investigation of GW190521's unique source property. In this paper, we set $G=1=c$ with the useful conversion factor $1 M_{\odot}=1.477 \mathrm{~km}=4.926 \times 10^{-6} \mathrm{~s}$. We also assume a "Planck" flat cosmology (when it is needed) with the Hubble constant $H_{0}=67.7 \mathrm{~km} \mathrm{~s}^{-1} \mathrm{Mpc}^{-1}$, and density parameters $\Omega_{M}=0.307$ and $\Omega_{\Lambda}=0.694$ [5]. 
Table 1. Summary of the source parameters of GW190521 as a quasicircular BBH merger, reported by the LIGO-Virgo collaboration [3] based on the BBH waveform model [6]. The symmetric $90 \%$ credible interval for each parameter is also quoted. Note that the parameters are written in our notation and masses are given in the source's rest frame; multiply by $(1+z)$ to convert to the observer frame.

\begin{tabular}{ccc}
\hline Parameter & Symbol & \\
\hline Primary mass $\left[M_{\odot}\right]$ & $m_{2}^{\mathrm{r}}$ & $85_{-14}^{+21}$ \\
Secondary mass $\left[M_{\odot}\right]$ & $m_{1}^{\mathrm{r}}$ & $66_{-18}^{+17}$ \\
Primary spin magnitude & $\left|\vec{\chi}_{2}\right|$ & $0.69_{-0.62}^{+0.27}$ \\
Secondary spin magnitude & $\left|\vec{\chi}_{1}\right|$ & $0.73_{-0.64}^{+0.24}$ \\
Total mass [M $\left.M_{\odot}\right]$ & $m_{t}^{\mathrm{r}}\left(=m_{1}^{\mathrm{r}}+m_{2}^{\mathrm{r}}\right)$ & $150_{-17}^{+29}$ \\
Mass ratio & $q\left(=m_{1}^{\mathrm{r}} / m_{2}^{\mathrm{r}} \leq 1\right)$ & $0.79_{-0.29}^{+0.19}$ \\
Luminosity Distance [Gpc] & $D_{L}$ & $5.3_{-2.6}^{+2.4}$ \\
Redshift & $z$ & $0.82_{-0.34}^{+0.28}$ \\
\hline
\end{tabular}

A key element to better understand GW190521 is the precise measurement of the binary parameters (see, for example, Ref. [7] for a possibility of an intermediate mass ratio inspiral). GW190521 is, however, a much heavier BBH system than previously observed GW events, and one of the difficulties here is the short duration and bandwidth of the GW signal that can be observed in the LIGO/Virgo band. In the case of the quasicircular BBH scenario (which is most favored by the LIGO/Virgo analysis [3,4]), the coalescing time and the number of GW cycles at frequency $f$ (in the observer frame) are estimated as:

$$
\begin{aligned}
t_{c} & \sim 1.3(1+z)^{-5 / 3}\left(\frac{m_{1}^{\mathrm{r}}}{66 M_{\odot}}\right)^{-1}\left(\frac{m_{2}^{\mathrm{r}}}{86 M_{\odot}}\right)^{-1}\left(\frac{m_{t}^{\mathrm{r}}}{150 M_{\odot}}\right)^{1 / 3}\left(\frac{f}{10.0 \mathrm{~Hz}}\right)^{-8 / 3} \mathrm{~s}, \\
N_{c} & \sim 1.0 \times 10^{1}(1+z)^{-5 / 3}\left(\frac{m_{1}^{\mathrm{r}}}{66 M_{\odot}}\right)^{-1}\left(\frac{m_{2}^{\mathrm{r}}}{85 M_{\odot}}\right)^{-1}\left(\frac{m_{t}^{\mathrm{r}}}{150 M_{\odot}}\right)^{1 / 3}\left(\frac{f}{10.0 \mathrm{~Hz}}\right)^{-5 / 3} .
\end{aligned}
$$

This indicates the lack of the GW signals from the sufficiently long inspiral phase. Because of the short duration signal dominated by the merger and ringdown phases, for example, only weak constraints are obtained for the component $\mathrm{BH}$ spins and their orientations [4]. Furthermore, even alternative interpretations of the observed GW signal other than massive quasicircular BBH merger in general relativity (GR) would become more relevant; several plausible scenarios are assessed in Section 6 of Ref. [4] by the LIGO-Virgo collaboration.

At the same time, the estimation in Equations (1) and (2) suggests a natural way to overcome the hurdle here: observe the inspiral GW signal in the low-frequency band offered by space-based GW detectors. Future GW astronomy in the 2030s will utilize the LISA observatory in the milli-Hz band [8] and deci-Hz GW detectors such as B-DECIGO [9], a prototype GW antenna of the DECIGO mission $[10,11]$ (other proposed GW missions in the low-frequency band, including Taiji [12] and TianQin [13] in the milli-Hz band and MAGIS [14] and TianGO [15] in the deci-Hz band, are concisely summarized in, for example, reviews by $\mathrm{Ni}[16,17])$. Figure 1 plots the track of the strain sensitivity curve of a GW190521-like non-spinning BBH system, assuming the quasicircular evolution and a simple inspiral-merger-ringdown (IMR) amplitude model given in Equation (16). At the GW frequency $f=0.1 \mathrm{~Hz}$, for example, Equations (1) and (2) give the coalescence time $t_{c} \sim 1.1 \times 10^{5} \mathrm{~s}$ and the number of GW cycles $N_{c} \sim 8.1 \times 10^{3}$ with the dimensionless characteristic strain:

$$
h_{\mathcal{c}} \sim 7.6 \times 10^{-22}(1+z)^{5 / 6}\left(\frac{\mathcal{M}^{\mathrm{r}}}{65.1 M_{\odot}}\right)^{5 / 6}\left(\frac{f}{0.1 \mathrm{~Hz}}\right)^{-1 / 6}\left(\frac{D_{L}}{5.3 \mathrm{Gpc}}\right)^{-1},
$$


where we introduce the source's rest-frame chirp mass $\mathcal{M}^{\mathrm{r}} \equiv v^{3 / 5} m_{t}^{\mathrm{r}}$ with the symmetric mass ratio $v \equiv m_{1}^{\mathrm{r}} m_{2}^{\mathrm{r}} / m_{t}^{\mathrm{r} 2}$. In fact, we find in Section 3 that the sky and polarization averaged signal-to-noise ratio (SNR) (whose meaning is momentarily clarified in Section 2) accumulated 5 years before the final coalescence would be $\sim 5.9 \times 10^{1}$ in the B-DECIGO band and $\sim 2.7$ in the LISA band. These estimations show that the early inspiral signal of GW190521-like BBHs would be sensitive in the LISA band and loud in the deci-Hz band.

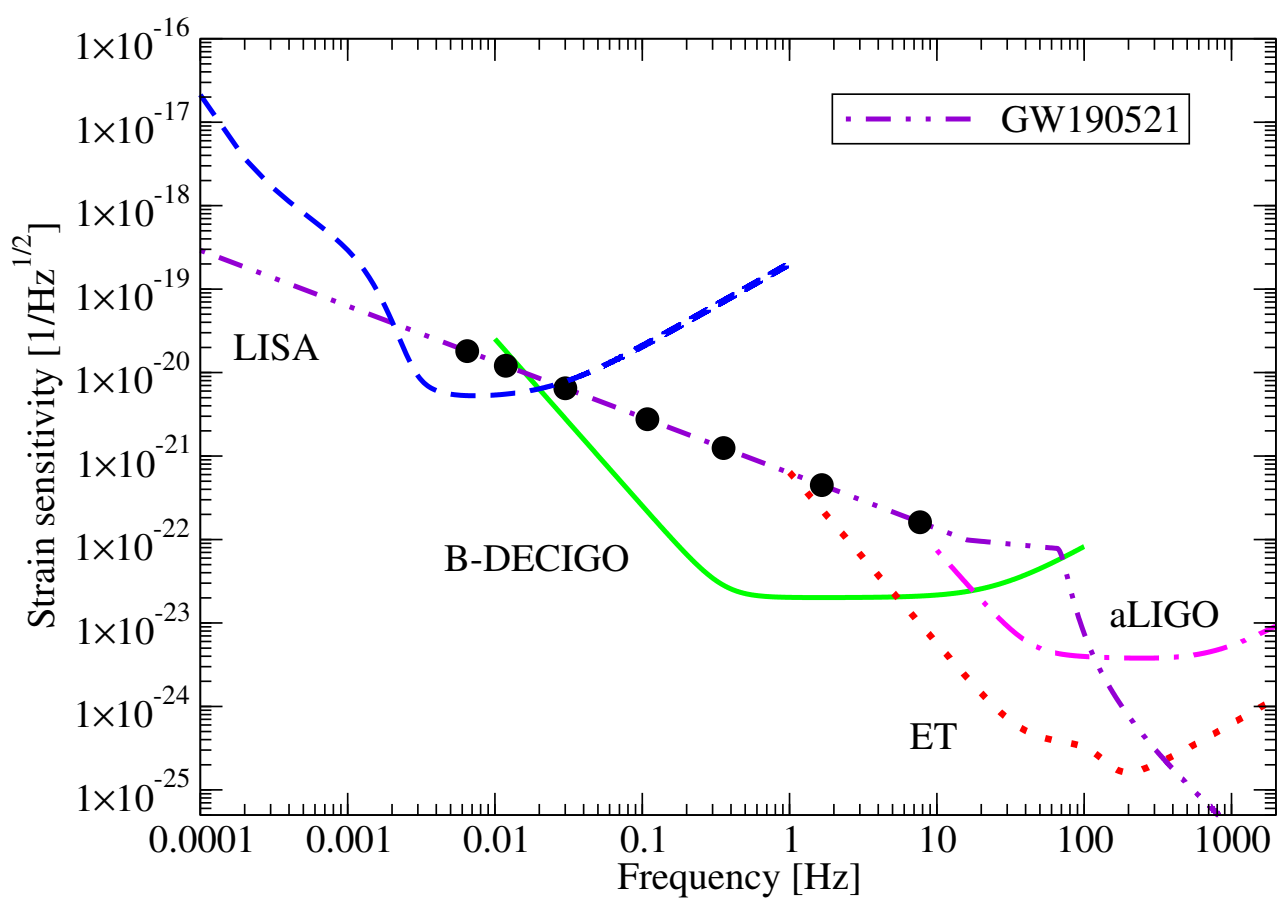

Figure 1. Strain sensitivity curves for the ground-based aLIGO and a next-generation (3G) detector: Einstein Telescope (ET) and space-based B-DECIGO and LISA, together with the GW amplitude of a GW190521-like nonprecessing, quasicircular BBH. We use the median values in Table 1 as source parameters. The noise power spectral density (PSD) for each GW detector is given in Section 2.1, and the spectral density of the BBH amplitude is obtained by the nonspinning, IMR amplitude model in Section 2.3. As a reference, we mark with the black dots with 5 years, 1 year, 1 month, 1 day, $1 \mathrm{~h}$, $1 \mathrm{~min}$, and $1 \mathrm{~s}$ before the merger time (1) from the left to the right.

Although the observation with LISA and B-DECIGO alone would provide valuable information on the inspiral GW signal from the GW190521-like BBH system, the true potential of having the low-frequency sensitivity will be revealed only when it is combined with the high-frequency sensitivity in the hecto-Hz band. As illustrated in Figure 1, the lateinspiral and merger-ringdown GW signals of the GW190521-like BBH are best detected with aLIGO, Virgo, and KAGRA [18]. In addition to ground-based GW observatories that are online, the next generation (3G) of ground-based detectors such as the Einstein Telescope (ET) [19,20] (see also Ref. [21] for Cosmic Explorer (CE)) will significantly improve the visibility of GW190521-like BBH systems. We will see in Section 3 that the averaged SNR of the late-inspiral and ringdown signals in the ET band would be $\sim 2.7 \times 10$ and $\sim 1.5 \times 10^{2}$, respectively. The joint "space + ground" observation across the full GW bands would be therefore the best way to observe the GW190521-like BBH systems; this is the basic idea of multiband GW astronomy.

Soon after the first detection of GW150914 [22], the potential of the multiband GW astronomy of BBH systems with LISA and aLIGO was emphasized [23] (see also Ref. [24]). This study was immediately followed up with more detailed analyses. The works include (but not limited to) the improved estimation of source parameters [25,26], tests of GR with high precision [27-32], refined event rate estimations [33], probing environment effects $[34,35]$, and new data analysis ideas [36]; these all prove the scientific values added 
by the multiband observation. Among these investigations, Refs. [9,37-41] demonstrated that the multiband observation of stellar-mass BBH systems will further benefit from having (B-)DECIGO in the deci-Hz band, which naturally bridges the gap between the LISA and aLIGO bands.

\section{Goals and Organization of This Paper}

Our purpose in this paper is to explore the prospects of the multiband observation of GW190521-like nonprecessing, quasicircular "intermediate-mass" BBHs. The possibility of the multiband observation of intermediate-mass BBHs with deci-Hz GW detectors was pointed out by Amaro-Seoane and Santamaria [42] and Yagi [43]. We consider these observations across the full GW spectrum provided by LISA in the milli-Hz band, BDECIGO in the deci-Hz band, and aLIGO and ET in the hecto-Hz, looking at two specific aspects of multiband GW astronomy/physics: parameter estimation errors and tests of GR.

We begin in Section 2 by providing our set of basic tools for the signal analysis in the matched filtering technique: (i) the noise PSD in Section 2.1, (ii) the GW signal models in Sections 2.2 and 2.3, and (iii) the multiband Fisher matrix formalism in Section 2.4. In Section 3, we present the parameter estimation errors of GW190521-like nonprecessing, quasicircular BBH systems using multiband GW observations. They are displayed in Table 2 and 3. Based on the estimated errors, we then examine in Section 4 to what extent the future multiband observations of GW190521-like BBH systems will improve tests of GR; this includes an inspiral-ringdown consistency test (see Table 4) and a simple test to discriminate between the remnant BH based on GR and other remnant compact objects (see Figure 2) We conclude in Section 5 with complications and various effects, which are not covered in this paper due to our assumptions and simplifications.

The appendices contain some additional analysis and information. While a quasicircular $\mathrm{BBH}$ merger is the most favored scenario in the LIGO/Virgo analysis [3,4], other alternative scenarios such as an eccentric BBH merger may also be consistent with the observed source for GW190521 [44]. We briefly discuss the multiband visibility of a GW190521-like eccentric BBH in Appendix A. Additionally, the additional noise PSDs of ground/space-based current/future GW detectors is provided in Appendix B as a complement to our treatment in Section 2.1.

Table 2. Parameter estimation errors of mass parameters $(m, v)$ and spin parameters $\left(\chi_{s}, \chi_{a}\right)$ for the GW190521-like quasicircular BBH inspiral, normalized to the total multiband SNR $\rho_{\text {tot }}$; for example, the result of "BD" in "BD + aLIGO" is normalized to $\rho_{\text {tot }}=5.93 \times 10^{1}$. We assume that the true binary parameters are given by the median values of Table 1 -i.e., $m_{t}=151.0 M_{\odot}, v=0.246, \chi_{s}=0.71$, and $\chi_{a}=0.02$. Since the source location can be determined well by B-DECIGO (BD here) [9,39], we fix $1+z=1.82$. Note that only the inspiral SNR of aLIGO is 1.76 , which is too small to give meaningful estimation errors. Additionally, any normalized estimation errors $\delta \hat{\boldsymbol{\theta}}>1.0 \times 10^{6}$ are discarded from this table.

\begin{tabular}{|c|c|c|c|c|c|}
\hline GW Detector & SNR & $\delta \hat{m}_{t} / m_{t}$ & $\delta \hat{v} / v$ & $\delta \hat{\chi}_{s} / \chi_{s}$ & $\delta \hat{\chi}_{a} / \chi_{a}$ \\
\hline \multicolumn{6}{|l|}{$\mathrm{BD}+\mathrm{aLIGO}$} \\
\hline $\mathrm{BD}$ & $5.92 \times 10^{1}$ & $3.51 \times 10^{-1}$ & $5.84 \times 10^{-1}$ & 4.91 & $1.12 \times 10^{3}$ \\
\hline aLIGO & 1.76 & $\cdots$ & $\ldots$ & $\cdots$ & $\cdots$ \\
\hline $\mathrm{BD}+\mathrm{aLIGO}$ & $5.93 \times 10^{1}$ & $3.44 \times 10^{-1}$ & $5.73 \times 10^{-1}$ & 4.73 & $1.08 \times 10^{3}$ \\
\hline \multicolumn{6}{|l|}{$\mathrm{BD}+\mathrm{ET}$} \\
\hline ET & $2.72 \times 10^{1}$ & $3.70 \times 10^{2}$ & $6.00 \times 10^{2}$ & $5.86 \times 10^{3}$ & \\
\hline $\mathrm{BD}+\mathrm{ET}$ & $6.52 \times 10^{1}$ & $2.88 \times 10^{-1}$ & $4.80 \times 10^{-1}$ & 3.07 & $7.01 \times 10^{2}$ \\
\hline \multicolumn{6}{|l|}{$\mathrm{LISA}+\mathrm{BD}+\mathrm{ET}$} \\
\hline LISA & 2.68 & $1.73 \times 10^{1}$ & $2.89 \times 10^{1}$ & $2.37 \times 10^{3}$ & 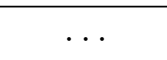 \\
\hline $\mathrm{LISA}+\mathrm{BD}$ & $5.93 \times 10^{1}$ & $1.83 \times 10^{-1}$ & $3.04 \times 10^{-1}$ & 4.01 & $9.11 \times 10^{2}$ \\
\hline $\mathrm{LISA}+\mathrm{BD}+\mathrm{ET}$ & $6.52 \times 10^{1}$ & $1.45 \times 10^{-1}$ & $2.41 \times 10^{-1}$ & 2.52 & $5.71 \times 10^{2}$ \\
\hline
\end{tabular}


Table 3. Parameter estimation errors of the central frequency $f_{c}$ and the quality factor $Q$ of the GW190521-like remnant BH using only the ringdown GW signal. The errors are normalized to the signal-to-noise ratio (SNR) of each corresponding detector.

\begin{tabular}{cccc}
\hline GW Detector & SNR & $\delta \hat{f}_{c} / f_{c}$ & $\delta \hat{Q} / Q$ \\
\hline aLIGO & $1.08 \times 10^{1}$ & $3.42 \times 10^{-1}$ & $2.71 \times 10^{-1}$ \\
ET & $1.47 \times 10^{2}$ & $3.66 \times 10^{-1}$ & $2.86 \times 10^{-1}$ \\
\hline
\end{tabular}

Table 4. Parameter estimation errors of the remnant mass and spin of GW190521-like and GW150914-like BBH systems, using only the ringdown GW signal as well as the inspiral GW signal inferred via the NR remnant fitting formulas; the best fit values are $\left(M_{f} / m, \chi_{f}\right) \sim(0.904,0.883)$ for the GW190521-like BBH (recall Equation (27)) and $\left(M_{f} / m, \chi_{f}\right) \sim(0.891,0.898)$ for the GW150914-like BBH. Here, BD is an abbreviation for B-DECIGO. The results in the single band are normalized to the

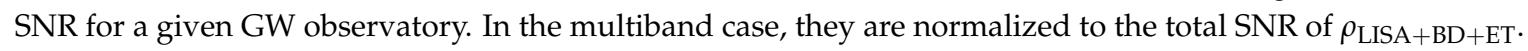

\begin{tabular}{|c|c|c|c|c|c|c|}
\hline \multirow[b]{2}{*}{ GW Detector } & \multicolumn{3}{|c|}{ GW190521-Like BBH } & \multicolumn{3}{|c|}{ GW150914-Like BBH } \\
\hline & SNR & $\delta M_{f} / M_{f}$ & $\delta \chi_{f} / \chi_{f}$ & SNR & $\delta M_{f} / M_{f}$ & $\delta \chi_{f} / \chi_{f}$ \\
\hline \multicolumn{7}{|c|}{ Single band: Ringdown GW signal } \\
\hline aLIGO & $1.08 \times 10^{1}$ & $3.09 \times 10^{-1}$ & $7.47 \times 10^{-1}$ & $1.78 \times 10^{1}$ & $3.05 \times 10^{-1}$ & $6.47 \times 10^{-1}$ \\
\hline ET & $1.47 \times 10^{2}$ & $3.31 \times 10^{-1}$ & $7.90 \times 10^{-1}$ & $2.66 \times 10^{2}$ & $3.26 \times 10^{-1}$ & $6.60 \times 10^{-1}$ \\
\hline \multicolumn{7}{|c|}{ Single band: inspiral GW signal } \\
\hline $\mathrm{BD}$ & $5.92 \times 10^{1}$ & $3.31 \times 10^{-1}$ & $9.63 \times 10^{-1}$ & $2.51 \times 10^{2}$ & $3.19 \times 10^{-1}$ & $9.43 \times 10^{-1}$ \\
\hline \multicolumn{7}{|c|}{ Multiband: inspiral GW signal } \\
\hline $\mathrm{BD}+\mathrm{ET}$ & $6.52 \times 10^{1}$ & $3.55 \times 10^{-1}$ & $5.91 \times 10^{-1}$ & $5.18 \times 10^{2}$ & $1.07 \times 10^{-1}$ & $4.48 \times 10^{-1}$ \\
\hline LISA + BD & $5.93 \times 10^{1}$ & $2.46 \times 10^{-1}$ & $8.05 \times 10^{-1}$ & $2.51 \times 10^{2}$ & $6.39 \times 10^{-1}$ & 1.85 \\
\hline $\mathrm{LISA}+\mathrm{BD}+\mathrm{ET}$ & $6.52 \times 10^{1}$ & $1.75 \times 10^{-1}$ & $4.98 \times 10^{-1}$ & $5.18 \times 10^{2}$ & $1.06 \times 10^{-1}$ & $4.33 \times 10^{-1}$ \\
\hline
\end{tabular}
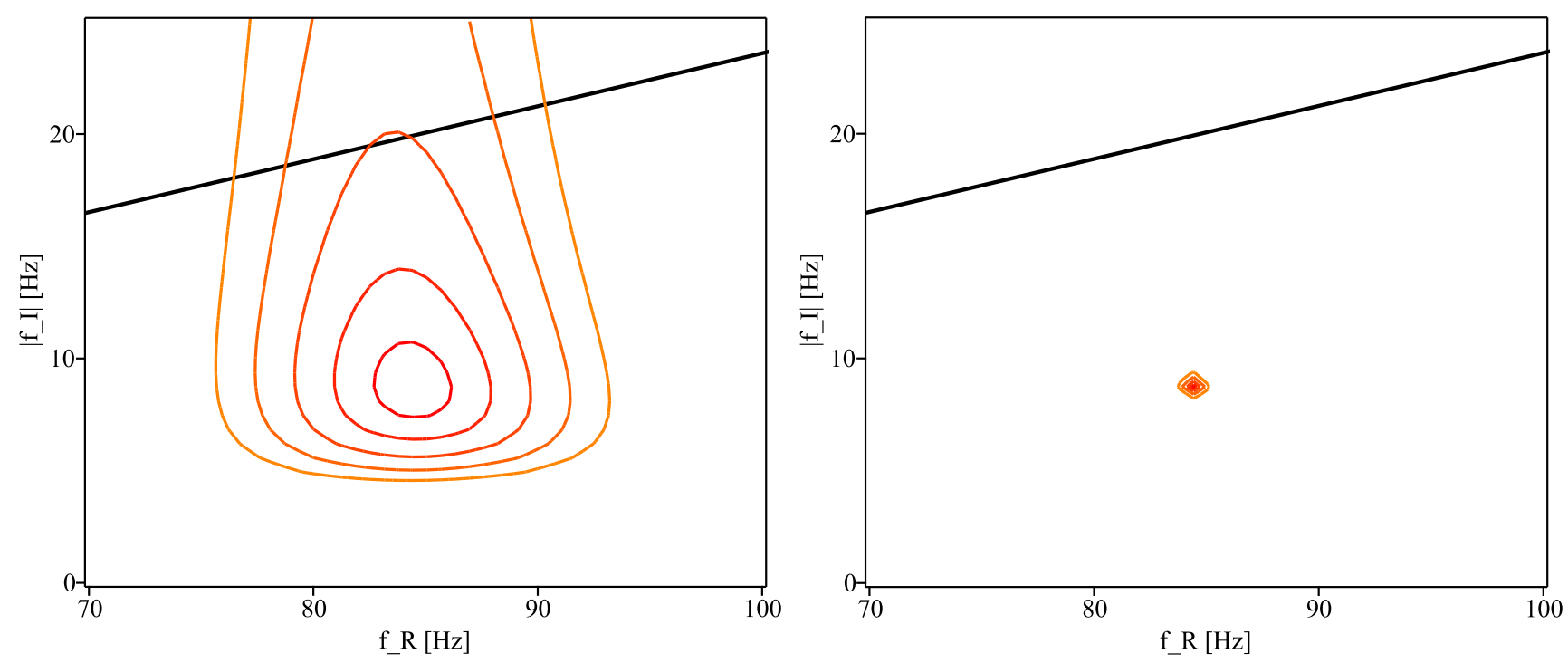

Figure 2. Simple remnant test in the $\left(f_{\mathrm{R}}, f_{\mathrm{I}}\right)$ plane. The QNM frequency is given in the observer frame, and we assume the GW190521-like ringdown GW signals with $\left(M_{f} / m, \chi_{f}\right) \sim(0.904,0.883)$ (27). The figure shows the parameter estimation error with the aLIGO noise curve (left, SNR $=1.08 \times 10^{1}$ ) and the ET one (right, SNR $=1.47 \times 10^{2}$ ). The black lines are the Schwarzschild limit of $\left|f_{\mathrm{I}}\right| / f_{\mathrm{R}} \approx 0.236$. The colored ellipses show the contours of $1 \sigma, 2 \sigma, 3 \sigma, 4 \sigma$, and $5 \sigma$. To obtain these two-dimensional plots, the time and phase parameters $\left(t_{0}, \phi_{0}\right)$ have been marginalized out. 
Throughout this paper, the binary parameters and GW frequencies measured in the source's rest frame are denoted with the index ' $r$ ', explicitly distinguished from those in the observer frame.

\section{Method for Signal-to-Noise Ratio and Fisher Analysis}

In this section, we summarize our methodology of multiband GW data analysis in the specific context of a nonprecessing, spinning, quasicircular GW190521-like BBH system, where the spins are aligned to the orbital angular momentum; an alternative scenarios of non-zero orbital eccentricity in GW190521-like BBH system will be discussed in Appendix A. Our simple framework here is largely based on the previous B-DECIGO works [9,38].

\section{1. aLIGO, ET, B-DECIGO and LISA}

For our multiband GW observation, we follow Ref. [38] by considering the four GW observatories: aLIGO and ET in the hecto-Hz band, B-DECIGO in the deci-Hz, and LISA in the milli-Hz band (see also Appendix B for some of other GW detectors in these bands). It should be noted that we shall use the "non sky-averaged" PSD $S_{n}(f)$; we will account for the average over the GW detector's antenna pattern function at the level of the waveform.

For aLIGO in the hecto-Hz band, we use Equation (4.7) in Ref. [45],

$$
\begin{aligned}
S_{n}^{\mathrm{aLIGO}} & =10^{-48}\left(0.0152 x^{-4}+0.2935 x^{9 / 4}+2.7951 x^{3 / 2}-6.5080 x^{3 / 4}+17.7622\right) \mathrm{Hz}^{-1} ; \\
x & =\frac{f}{245.4 \mathrm{~Hz}} .
\end{aligned}
$$

For a third generation (3G) GW interferometer-ET in the hecto-Hz band-we find Table 1 in Ref. [46] as:

$$
\begin{aligned}
S_{n}^{\mathrm{ET}}= & 1.5 \times 10^{-52}\left(y^{-4.1}+186.0 y^{-0.69}\right. \\
& \left.+233.0 \times \frac{1.0+31.0 y-65.0 y^{2}+52.0 y^{3}-42.0 y^{4}+10.0 y^{5}+12.0 y^{6}}{1.0+14.0 y-37.0 y^{2}+19.0 y^{3}+27.0 y^{4}}\right) \mathrm{Hz}^{-1} ; \\
y= & \frac{f}{200.0 \mathrm{~Hz}} .
\end{aligned}
$$

For B-DECIGO in the deci-Hz band, we use Equation (20) in Ref. [38], originally proposed by Nakamura et al. [9]:

$$
\begin{aligned}
S_{n}^{\mathrm{BD}} & =\left[\left(2.01 \times 10^{-23}\right)^{2}+\left(\frac{2.53 \times 10^{-18}}{F^{2}}\right)^{2}+\left(8.00 \times 10^{-22} \mathrm{~F}\right)^{2}\right] \mathrm{Hz}^{-1} ; \\
F & =\frac{f}{1.0 \times 10^{-3} \mathrm{~Hz}} .
\end{aligned}
$$

For LISA in the milli-Hz band, we use Equation (1) of Ref. [47], which is based on the 2018 LISA Phase-0 reference design parameters. It reads:

$$
\begin{aligned}
S_{n}^{\mathrm{LISA}}= & {\left[\left(2.4602 \times 10^{-41}+4.0504 \times 10^{-38} z^{2}+\frac{4.7850 \times 10^{-48}}{z^{2}}\right.\right.} \\
& \left.\left.+\frac{2.8485 \times 10^{-51}}{z^{4}}+\frac{3.9412 \times 10^{-58}}{z^{6}}\right)+S_{c}^{\mathrm{LISA}}\right] \mathrm{Hz}^{-1} ; \\
z= & \frac{f}{1.0 \mathrm{~Hz}} .
\end{aligned}
$$

where $S_{c}^{\mathrm{LISA}}(f)$ represents the effective PSD due to the unresolved galactic binaries; the explicit expression is given in Equation (14) of Ref. [47], and we have assumed a 4-year mission for Figure 1. Here, our expression $S_{n}^{\text {LISA }}(7)$ is smaller than Equation (1) in Ref. [47] 
by a overall factor of 5; the difference arises simply because Equation (7) does not account for the sky-averaging.

\subsection{Waveform Models}

We employ as our BBH waveform model the frequency domain, "restricted" waveform in the stationary phase approximation for the inspiral phase, and the frequency domain, single-mode waveform for the ringdown phase. We shall restrict our waveform model to these two phases to simplify our analysis as far as possible; the complete IMR treatment at the level of waveform (using, for example, the "effective-one-body" (EOB) approach [48,49] and the "phenomenological" (IMRPhenom) model $[50,51]$ ) will be left for future work.

The GW waveform from a BBH inspiral in the frequency domain has the well-known form (see, for example, Ref. [52]):

$$
\tilde{h}_{\text {Insp }}(f)=\mathcal{A} f^{-7 / 6} e^{i \Psi_{\text {Insp }}(f)},
$$

where $\mathcal{A}$ is the "Newtonian" amplitude averaged over all sky positions and binary orientations (see, for example, Ref. [53]), so that:

$$
\mathcal{A} \equiv \frac{2}{5} \sqrt{\frac{5}{24}} \pi^{-2 / 3} \frac{\mathcal{M}^{5 / 6}}{D_{L}} .
$$

The waveform's frequency domain phase, $\Psi_{\text {Insp }}(f)$, in the post-Newtonian (PN) approximation is given by (see, for example, Ref. [54]):

$$
\Psi_{\text {Insp }}(f)=2 \pi f t_{c}-\Psi_{c}-\frac{\pi}{4}+\frac{3}{128 v v^{5}}\left(\Delta \Psi_{3.5 \mathrm{PN}}^{\mathrm{pp}}+\Delta \Psi_{3.5 \mathrm{PN}}^{\mathrm{pp}-\text { spin }}+\Delta \Psi_{3.5 \mathrm{PN}}^{\mathrm{BH}-\text { tidal }}\right),
$$

where $v \equiv\left(\pi m_{t} f\right)^{1 / 3}$ is the PN parameter (in terms of the observer-frame total mass), and $t_{c}$ and $\Psi_{c}$ are the time and phase at coalescence. The phase terms $\Delta \Psi_{3.5 \mathrm{PN}}^{\mathrm{pp}}$ and $\Delta \Psi_{3.5 \mathrm{PN}}^{\mathrm{pp}-\text { spin }}$ are the 3.5PN spin-independent, point-particle contributions derived in Ref. [55] and the 3.5PN spin-dependent, point-particle contributions that include linear spin-orbit [56,57], quadratic-in-spin [58], and cubic-in-spin [59] effects, respectively. The remaining phase term $\Delta \Psi_{3.5 \mathrm{PN}}^{\mathrm{BH} \text {-tidal }}$ is related to the tidal response of a spinning $\mathrm{BH}$ as a finite-size body-i.e., $\mathrm{BH}$-absorption corrections such as the GW energy and angular momentum fluxes down to the horizons and the associated evolution of the BH itself [60-62].

Meanwhile, limited to only fundamental $(n=0), \ell=2=m$ mode, the time-domain, single-mode ringdown waveform measured at a GW observatory is written as [63-65]:

$$
h_{\text {Ring }}\left(f_{c}, Q, t_{0}, \phi_{0} ; t\right)= \begin{cases}e^{-\frac{\pi f_{c}\left(t-t_{0}\right)}{Q} \cos \left[2 \pi f_{c}\left(t-t_{0}\right)-\phi_{0}\right]} & \text { for } t \geq t_{0} \\ 0 & \text { for } t<t_{0}\end{cases}
$$

where $t_{0}$ and $\phi_{0}$ are the initial time and phase of the ringdown, respectively, and we have ignored the overall amplitude so that Equation (11) is not normalized; the initial ringdown amplitude may be determined by matching the ringdown GW waveform with the merger one (refer to, for example, Equation (16) below). When the final remnant object is a Kerr $\mathrm{BH}$, the central frequency $f_{c}$ and the quality factor $Q$ are given in terms of quasinormal mode $(\mathrm{QNM})$ frequencies $\left(f_{\mathrm{QNM}}=f_{\mathrm{R}}+i f_{\mathrm{I}}\right)$ of the remnant $\mathrm{BH}$ as (see Equation (7) of Ref. [66]):

$$
f_{\mathcal{c}}:=f_{\mathrm{R}}, \quad Q:=-\frac{f_{\mathrm{R}}}{2 f_{\mathrm{I}}}
$$


It should be noted that $f_{\mathrm{QNM}}=f_{\mathrm{QNM}}^{\mathrm{r}}(1+z)^{-1}$ here is given in the observer frame. The Fourier transforms of Equation (11) provide the corresponding frequency-domain waveform, which takes the form (here we follow the convention of Ref. [63]):

$$
\tilde{h}_{\text {Ring }}\left(f_{c}, Q, t_{0}, \phi_{0} ; f\right)=\frac{Q\left(f_{c} \cos \phi_{0}-2 i Q f \cos \phi_{0}+2 Q f_{c} \sin \phi_{0}\right)}{\pi\left(f_{c}+2 i Q f_{c}-2 i Q f\right)\left(f_{c}-2 i Q f_{c}-2 i Q f\right)} \mathrm{e}^{2 i \pi f t_{0}} .
$$

We note that this frequency-domain GW waveform is not normalized either, following the approach presented in Appendix B of Ref. [64]. The maximized SNR over the initial ringdown phase $\phi_{0}$ is discussed in Ref. [67].

The ringdown waveforms (11) and (13) require input data for the QNM frequency of the final remnant $\mathrm{BH}$. Because we do not consider the complete IMR phase at the level of waveforms, we employ the numerical relativity (NR) remnant-fitting formulas (see, for example, Refs. [68-73] and references therein), from which the final mass $M_{f}$ and spin $\mathbf{S}_{f}$ of the remnant $\mathrm{BH}$ are consistently inferred for a given initial $\mathrm{BH}$ masses $m_{1,2}$ and dimensionless spin parameters $\vec{\chi}_{1,2}$ (in the inspiral phase) as:

$$
M_{f}=M_{f}\left(m_{1}, m_{2}, \vec{\chi}_{1}, \vec{\chi}_{2}\right), \quad \chi_{f} \equiv \frac{\left|\mathbf{S}_{f}\right|}{M_{f}^{2}}=\chi_{f}\left(m_{1}, m_{2}, \vec{\chi}_{1}, \vec{\chi}_{2}\right) .
$$

The mass and spin of the remnant $\mathrm{BH}$ in the remnant formulas are derived in the isolated horizon framework (see, for example, Ref. [74]), not obtained from the ringdown GWs. The latter is used only for checking the internal consistency of the formulas.

We then generate the accurate numerical data of $f_{\mathrm{QNM}}$ of the inferred remnant $\mathrm{BH}$ with the Black Hole Perturbation Club (B.H.P.C.) code [75] to obtain $f_{c}$ and $Q$ (for accurate numerical data of $f_{\mathrm{QNM}}$, see also Ref. [76], Emanuele Berti's "Ringdown" website [77], Ref. [78], and the Black Hole Perturbation Toolkit [79]). In practice, it is also convenient to present $f_{c}$ and $Q$ by means of a compact analytical formula. Such a formula for the $(\ell=2, m=2, n=0)$ mode is obtained in Ref. [65] (by performing fits to the numerical QNM frequency data), and it reads:

$$
f_{c}=\frac{1}{2 \pi M_{f}}\left[f_{1}+f_{2}\left(1-\chi_{f}\right)^{f_{3}}\right], \quad Q=q_{1}+q_{2}\left(1-\chi_{f}\right)^{q_{3}},
$$

with $f_{1}=1.5251, f_{2}=-1.1568, f_{3}=0.1292, q_{1}=0.7000, q_{2}=1.4187$ and $q_{3}=-0.4990$.

\subsection{Signal-to-Noise Ratio}

We estimate the SNR of a complete IMR GW signal, making use of the simple frequency-domain, IMR "amplitude" model [9]. This model is motivated by the waveform amplitude in the IMRPhenomB model [80] and it is given by:

$$
\operatorname{IMR}(f)=\mathcal{A} \times \begin{cases}f^{-7 / 6} & \text { for } f<f_{\max }, \\ f_{\max }^{-1 / 2} f^{-2 / 3} & \text { for } f_{\max } \leq f<f_{\mathrm{R}}^{\mathrm{r}} /(1+z), \\ \frac{f_{\max }^{-1 / 2}\left[f_{\mathrm{R}}^{\mathrm{r}} /(1+z)\right]^{-2 / 3}\left[f_{\mathrm{I}}^{\mathrm{r}} /(1+z)\right]^{2}}{\left\{f-\left[f_{\mathrm{R}}^{\mathrm{r}} /(1+z)\right]\right\}^{2}+\left[f_{\mathrm{I}}^{\mathrm{r}} /(1+z)\right]^{2}} & \text { for } f_{\mathrm{R}}^{\mathrm{r}} /(1+z) \leq f,\end{cases}
$$

where the overall constant $\mathcal{A}$ is chosen to be the (averaged) GW signal's amplitudes in the inspiral phase (9). We set $f_{\max }=1 /\left[6^{3 / 2} \pi(1+z) m_{t}^{\mathrm{r}}\right]$ as the GW frequency at the innermost stable circular orbit (ISCO) of a test particle in the Schwarzschild spacetime with the total mass of $\mathrm{BBH} m_{t}^{\mathrm{r}}$, and $f_{\mathrm{R}}^{\mathrm{r}}$ and $f_{\mathrm{I}}^{\mathrm{r}}$ are the real and imaginary parts of the QNM frequency (i.e., $\left.f_{\mathrm{QNM}}^{\mathrm{r}}=f_{\mathrm{R}}^{\mathrm{r}}+i f_{\mathrm{I}}^{\mathrm{r}}\right)$ of the fundamental $(n=0), \ell=2=m$ mode, which are determined by the final mass and spin of the remnant $\mathrm{BH}$; the redshift dependence appears because the model parameters $\left(m_{t}^{\mathrm{r}}, f_{\mathrm{R}}^{\mathrm{r}}, f_{\mathrm{I}}^{\mathrm{r}}\right)$ are all given in the source's rest frame (while the GW 
frequencies $f$ and $f_{\max }$ are in the observer frame). The averaged SNR (in the above sense) can be then obtained by

$$
\rho_{\text {ave }}=2\left\{\int_{f_{\text {in }}}^{f_{\text {end }}} \frac{[\operatorname{IMR}(f)]^{2}}{S_{n}(f)} d f\right\}^{1 / 2},
$$

where $S_{n}(f)$ is the noise PSD and the frequency range $\left[f_{\text {in }}, f_{\text {end }}\right]$ is determined by the GW detector with which we observe the GW signals. For the given Fourier transform of a GW signal $\tilde{h}(f)$, the SNR can be written in terms of either $\tilde{h}(f)$ itself with the dimension of $1 / \mathrm{Hz}$, the spectral density of the source amplitude $\sqrt{S_{h}(f)} \equiv 2 \sqrt{f}|\tilde{h}(f)|$ with the dimension of $1 / \sqrt{\mathrm{Hz}}$, or the dimensionless characteristic strain $h_{c}(f) \equiv 2 f|\tilde{h}(f)|$ [81]:

$$
\rho_{\text {ave }}=\left(\int_{f_{\text {in }}}^{f_{\text {end }}} \frac{|2 \tilde{h}(f)|^{2}}{S_{n}(f)} d f\right)^{1 / 2}=\left(\int_{f_{\text {in }}}^{f_{\text {end }}} \frac{\left|\sqrt{S_{h}(f)}\right|^{2}}{S_{n}(f)} \frac{d f}{f}\right)^{1 / 2}=\left(\int_{f_{\text {in }}}^{f_{\text {end }}} \frac{\left[h_{c}(f)\right]^{2}}{f S_{n}(f)} \frac{d f}{f}\right)^{1 / 2} .
$$

Note that the amplitude spectral density (i.e., the square root of the PSD of the source amplitudes) is $\sqrt{S_{h}(f)} \equiv 2 \sqrt{f}|\operatorname{IMR}(f)|$ in this model, and its track for the GW190521-like BBH is plotted in Figure 1 with the (square root of) the noise PSD $\sqrt{S_{n}(f)}$, assuming the median values of Table 1 and the remnant formulas of Ref. [68].

\subsection{Multiband Fisher Analysis}

We approximate the variance (i.e., uncertainty squared) associated with the measurement of a set of signal parameters, making use of the standard Fisher matrix formalism. The Fisher information matrix for a single-band GW detector is defined by:

$$
\left.\Gamma_{a b} \equiv\left(\frac{\partial \tilde{h}}{\partial \theta_{a}} \mid \frac{\partial \tilde{h}}{\partial \theta_{b}}\right)\right|_{\theta=\theta_{0}^{\prime}}
$$

where $\tilde{h}(f, \boldsymbol{\theta})$ is the frequency-domain GW signal described by the set of parameters $\boldsymbol{\theta}$, and $\boldsymbol{\theta}_{0}$ are the best-fit values of the binary parameters. The bracket defines the noise-weighted inner product over the frequency range of $\left[f_{\text {in }}, f_{\text {end }}\right][82]$ :

$$
(a \mid b) \equiv 2 \int_{f_{\text {in }}}^{f_{\text {end }}} \frac{\tilde{a}^{*}(f) \tilde{b}(f)+\tilde{b}^{*}(f) \tilde{a}(f)}{S_{n}(f)} d f
$$

where an asterisk ' $*$ ' denotes the complex conjugation. The inverse Fisher matrix defines the corresponding variance-covariance matrix $\Sigma^{a b} \equiv\left(\Gamma_{a b}\right)^{-1}$. In the limit of suitably high SNR [83], the variance of the parameter $\theta^{a}$ is given by:

$$
\sigma_{a}^{2}=\Sigma^{a a}
$$

In the case of the multiband analysis (to combine the information from, for example, aLIGO + B-DECIGO), we simply construct a multiband SNR and Fisher matrix by adding individual (averaged) SNRs and Fisher information matrices for each GW detector:

$$
\rho_{\text {tot }}^{2} \equiv \sum_{I}\left(\rho_{\text {ave }}^{I}\right)^{2}, \quad \Gamma_{a b}^{\mathrm{tot}} \equiv \sum_{I} \Gamma_{a b}^{I}
$$

where $\rho_{\mathrm{ave}}^{I}$ and $\Gamma_{a b}^{I}$ are the averaged SNR and the Fisher matrix for the $I$-th detector. The multiband variance-covariance matrix is defined by:

$$
\Sigma_{\text {tot }}^{a b} \equiv\left(\Gamma_{a b}^{\text {tot }}\right)^{-1},
$$

The variance of $\theta^{a}$ is then obtained by $\sigma_{a}^{2}=\Sigma_{\text {tot }}^{a a}$. 


\section{Parameter Estimation Errors via Multiband Observation}

In this section, we summarize the parameter estimation errors of the inspiral and ringdown phases for a nonprecessing, spinning, GW190521-like BBH system using the multiband GW network (LISA, B-DECIGO, aLIGO, and ET) detailed in Section 2.1. We follow Refs. [38,66] in our treatment of the Fisher matrix calculation for BBH GW signals (the setup here is slightly different from Ref. [38]; (i) we will assume a $5 \mathrm{yr}$ observation rather than a 4 yr observation and (ii) we will use the new LISA sensitivity curve proposed by Ref. [47] and displayed in Equation (7), not the earlier eLISA sensitivity curve presented in Ref. [84]). We will also continue to neglect the contribution from the merger GW signal (using our IMR amplitude model in Equation (16), we have the (averaged) merger SNR of 17.0, $1.97 \times 10^{2}$, and 3.42 for aLIGO, ET, and B-DECIGO, respectively, and the addition of these contributions to the signal analysis will improve the parameter estimations), for the reason that one cannot separate the inspiral, merger, and ringdown phases cleanly in the strict sense, although we have presented a simple IMR amplitude model in Equation (16). When the merger contribution is introduced into the inspiral or ringdown signal analysis, it causes some bias in the inspiral or ringdown parameter estimation. For example, the merger-ringdown waveform is parametrized by binary parameters, not solely by the remnant $\mathrm{BH}$ parameters after the merger. Similarly, in the inspiral-merger waveform, the "late" merger phase can be described by the overtones of QNMs [85]-i.e., the remnant $\mathrm{BH}$ parameters. Thus, we shall perform a inspiral-ringdown consistency test of GR without the merger contribution in Section 4.1.

\subsection{Setup of Fisher Analysis}

We set the default frequency interval of each GW detector $\left[f_{\text {low }}, f_{\text {up }}\right]$ as $[10.0,3.0 \times$ $\left.10^{3}\right] \mathrm{Hz}(\mathrm{aLIGO}),\left[2.0,3.0 \times 10^{3}\right] \mathrm{Hz}(\mathrm{ET}),\left[0.01,1.0 \times 10^{2}\right] \mathrm{Hz}(\mathrm{B}-\mathrm{DECIGO})$, and $[1.0 \times$ $\left.10^{-4}, 1.0\right] \mathrm{Hz}$ (LISA), respectively. We shall adopt the $T_{\mathrm{obs}}=5$-year observation time and assume that the binary merges at the GW frequency of the Schwarzschild ISCO, $f_{\text {ISCO }}=1 /\left[6^{3 / 2} \pi(1+z) m_{t}^{\mathrm{r}}\right]$. In this setup, the minimum frequency of the GW signal is:

$$
f_{\min }=9.24 \times 10^{-3}(1+z)^{-5 / 8}\left(\frac{\mathcal{M}^{\mathrm{r}}}{65.1 M_{\odot}}\right)^{5 / 8}\left(\frac{5 \mathrm{yr}}{T_{\mathrm{obs}}}\right)^{3 / 8} \mathrm{~Hz},
$$

where $\mathcal{M}^{\mathrm{r}}$ is the chirp mass in the source's rest frame, normalized to that of GW190521. Therefore, the GW signal observed by each GW detector is truncated at the corresponding initial frequency $f_{\text {in }} \equiv \max \left(f_{\min }, f_{\text {low }}\right)$ as well as the end frequency $f_{\text {end }} \equiv \min \left(f_{\text {ISCO }}, f_{\text {up }}\right)$; recall Figure 1.

The parameters of the inspiral waveform (8) are:

$$
\boldsymbol{\theta}_{\text {Insp }}=\left(f_{0} t_{c}, \Psi_{c}, \ln m_{t}, v, \chi_{s}, \chi_{a}\right),
$$

where we define the symmetric and anti-symmetric combinations of BH spins by $\chi_{s} \equiv$ $\left(\chi_{1}+\chi_{2}\right) / 2$ and $\chi_{a} \equiv\left(\chi_{1}-\chi_{2}\right) / 2$ with the component (aligned) BH spins $\chi_{1,2} \equiv\left|\vec{\chi}_{1,2}\right| \equiv$ $\left|\mathbf{S}_{1,2}\right| / m_{1,2}^{2}$. At the same time, the parameters of the ringdown waveform (13) are:

$$
\boldsymbol{\theta}_{\text {Ring }}=\left(t_{0}, \phi_{0}, f_{c}, Q\right) .
$$

It should be noted that the amplitude parameters are left out from the set of our independent parameters in both Equations (24) and (25). They are entirely uncorrelated with other parameters $\theta_{a}$ because the variance-covariance matrix $\Sigma^{a b}$ gives the variance $\sigma_{\ln \mathcal{A}}^{2}=\rho_{\text {ave }}^{-2}$ and the correlation $c^{\ln \mathcal{A}, a} \equiv \Sigma^{\ln \mathcal{A}, a} /\left(\sigma_{\ln \mathcal{A}} \sigma_{a}\right)=0$ for the inspiral GW signal (8) (see, for example, Ref. [86]), and similar for the ringdown GW signal (if we explicitly introduce the amplitude to the normalized waveform of Equation (13)). For simplicity, we consider that all (other) parameters are unconstrained. 
The best fit values of inspiral parameters are given by the median values in Table 1 with $t_{c}=0.0=\Psi_{c}$, while those of the ringdown parameters are assumed to be $\phi_{0}=0.0=t_{0}$ and:

$$
\left(f_{c}, Q\right)=(85.061 \mathrm{~Hz}, 4.8354),
$$

for the (observer-frame) central frequency and quality factor. Note here that the parameter estimation errors are independent of the value of the initial time $t_{0}$. In our frequencydomain, single-mode ringdown waveform in Equation (13), the $t_{0}$ dependence is factorized as $\mathrm{e}^{2 i \pi f t_{0}}$ and it does not contribute to the noise-weighted inner product in Equation (19). On the other hand, the estimation errors depend on the initial phase $\phi_{0}$ weakly [64].

The values in Equation (26) are obtained via Equation (12) for the given QNM frequency data of the remnant $\mathrm{BH}$, assuming that the remnant mass $M_{f}$ and spin $\chi_{f}$ of the final $\mathrm{BH}$ are inferred via the remnant formula in Equation (14) with the parameters of each component BH in Table 1. Specifically, we use the remnant formula provided by Ref. [71], and we quote:

$$
\left(\frac{M_{f}}{m_{t}}, \chi_{f}\right)=(0.90356,0.88269) \text {. }
$$

Note that these values are different from the mass and $\operatorname{spin}\left(M_{f}, \chi_{f}\right)=\left(142_{-16}^{+28} M_{\odot}\right.$, $\left.0.72_{-0.12}^{+0.09}\right)$ of the remnant BH reported in the LIGO/Virgo GW190521 detection paper [3]; we have assumed that the individual spins of GW190521-like BBH are nonprecessing and completely aligned to the orbital angular momentum for simplicity, while the observed GW190521 is actually considered to be a precessing BBH.

The associated QNM frequency data of this remnant $\mathrm{BH}$ are then generated by the B.H.P.C. code [75], yielding the results in Equation (26).

The root-mean-square of parameter estimation error scales like the inverse of SNR, $\sim 1 / \rho$, and it depends on both the $\rho$ and bandwidth over which the SNR is accumulated. To see the benefit of having wider bandwidth due to the multiband observation, we finally introduce the normalized root-mean-square error as:

$$
\delta \hat{\boldsymbol{\theta}} \equiv \rho \sigma,
$$

We display our error estimations in terms of $\delta \hat{\boldsymbol{\theta}}$ with the total averaged SNR $\rho_{\text {ave }}^{\text {tot }}$ accumulated over the multi-frequency bands.

\subsection{Result: Inspiral Phase}

In Table 2, we present the parameter estimation errors of mass parameters $(m, v)$ and spin parameters $\left(\chi_{s}, \chi_{a}\right)$ for the GW190521-like BBH inspiral in various combinations of ground/space-based GW observatories (while suppressing those of $t_{c}$ and $\Psi_{c}$ ). Here, it should be noted that only the inspiral phase is analyzed, and the merger-ringdown phase which contributes to the SNR for ground-based observatories is ignored; recall the merger SNR shown in Section 3. Therefore, the SNR for aLIGO quoted here is much smaller than the observed LIGO/Virgo network SNR of 14.5 [3].

In the single-band case, as seen in Figure 1, the inspiral GW signal of the GW190521like $\mathrm{BBH}$ is best observed by B-DECIGO because it can cover both the early (1 yr before merger) and late (around the ISCO) phases. However, B-DECIGO observation alone is not enough benefit to discern the spin parameters.

In the multiband cases, thanks to the wider bandwidth, the observation with BDECIGO and ET gives a factor of 2 improvement in all the parameter estimation, even if we observe only the inspiral GW signal. This is further refined if we combine the data from LISA, forming a triple-band network (although the estimated LISA SNR of $\sim 2.68$ is likely too small compared to the 'realistic' detection SNR threshold of $\sim 15$ [87]). Importantly, the normalized errors of $\mathrm{BH}$ spins $\left(\delta \hat{\chi}_{s} / \chi_{s}, \delta \hat{\chi}_{a} / \chi_{a}\right)=\left(2.52,5.71 \times 10^{2}\right)$ in this best case imply that the magnitudes of individual BH spins $\sigma_{\chi}$ can be recovered with the fractional 
statistical errors (i.e., now accounting for the total multiband SNR of $\rho_{\text {ave }}^{\text {tot }}=6.52 \times 10^{1}$ to the uncertainties; recall Equation (28)) $\left(\sigma_{\chi_{1}} / \chi_{1}, \sigma_{\chi_{2}} / \chi_{2}\right)=\left(2.43 \times 10^{-1}, 2.51 \times 10^{-1}\right)$.

The joint LISA, B-DECIGO, and ET observatories would be only viable network to measure all the binary parameters of GW190521-like BBH system in the inspiral phase, including BH's component spins.

\subsection{Result: Ringdown Phase}

In Table 3, we show the parameter estimation errors of the central frequency $f_{c}$ and quality factor $Q$ (while suppressing those of $t_{0}$ and $\phi_{0}$ ) for the ringdown phase of the GW190521-like BBH in ground-based observatories. For simplicity, we estimate the ringdown amplitude and associated SNR via the IMR amplitude model in Equation (16) (we should note that this approximation is likely too raw because the ringdown amplitude strongly depends on the starting time $t_{0}$ of the ringdown phase, which is difficult to determine in practice). We see that ET will be able to measure the QNM frequency of the remnant $\mathrm{BH}$ with the statistical error $\sim 10^{-3}$. At the same time, however, the difference in the normalized errors $\delta \hat{f}_{c}$ and $\delta \hat{Q}$ between the aLIGO and ET observations is not so evident. We speculate that this arises from the difference in the spectrum shapes (not the overall amplitudes) because the ringdown waveform (13) is narrow-banded in the frequency domain.

\section{The Implications for Tests of GR via Multiband Observation}

In this section, we explore to what extent the multiband observation of the GW190521like BBH system discussed in Section 3 could improve tests of GR. A handful of tests have been already formulated and performed with merging BBH systems (see, for example, Refs. [88-91]), and we follow the (very) simple tests proposed by Nakano et al. [66,92].

\subsection{A Consistency test of GR with the Inspiral and Ringdown GW Signals}

One possible test of GR with a BBH system is to establish the consistency of the mass and spin of the final remnant $\mathrm{BH}$ determined by two different parts of the GW signals. Thanks to the recent advancement in NR simulations of BBH systems [93-95] (see also Refs. [96-100]), one can infer these values from the initial component masses and spins measured from the inspiral GW signal (in the low-frequency band), making use of the NR fitting formulas for the remnant properties of the final $\mathrm{BH}$; recall Section 2.2. At the same time, they are directly estimated from the succeeding merger-ringdown GW signal (in the high-frequency band). This type of test is now known as the "IMR consistency test" [101,102]. By formulation, multiband observations of heavy BBH mergers such as GW190521-like BBH systems will be "golden binaries" [103] of such an IMR consistency test.

Given that the early inspiral and late ringdown GW signals will be best observed in a different frequency band (such as "B-DECIGO + aLIGO" network etc.), we here perform the multiband version of the "inspiral-ringdown consistency test" formulated by Nakano et al. [66] (see also Refs. [103,104]), solely using the inspiral and ringdown parts, and test the consistency of GR across the merger part, which is a highly dynamical phase in a strong-field regime. We estimate the statistical errors on $M_{f}$ and $\chi_{f}$ from the inspiral GW signal by using the (normalized) statistical errors $\delta \hat{\boldsymbol{\theta}}$ in Table 2 and applying a standard variance propagation of non-linear functions to the specific NR remnant formulas ("UIB formulas") [71] publicly available in LALInference [105,106]. Here, we ignore the systematic bias due to our specific choice of the NR remnant formulas, for simplicity (see, for example, Refs. $[99,102]$ for details). The errors from the ringdown GW signal are estimated from Table 3 , through the dependence of $f_{c}$ and $Q$ on $\left(M_{f}, \chi_{f}\right)$. Technically, this procedure requires the evaluation of the partial derivatives $\left(\partial f_{c} / \partial \chi_{f}\right)_{M_{f}}$ etc. to compute the variance propagation. We construct the numerical function of QNM frequencies $M_{f} f_{\mathrm{QNM}}\left(\chi_{f}\right)$ with the B.H.P.C. code [75] around the best fit values of Equation (27), from which these derivatives are extracted. 
In Table 4 , we present the parameter estimation errors of the remnant mass $M_{f}$ and spin $\chi_{f}$ from both the inspiral and ringdown phases. For the reference, we also present the same errors of the GW150914-like BBH with the redshifted component masses $\left(m_{1}, m_{2}\right)=\left(30 M_{\odot}, 40 M_{\odot}\right)$, spin magnitudes $\left(\chi_{1}, \chi_{2}\right)=(0.9,0.7)$, and the luminosity distance $D_{L}=0.4 \mathrm{Gpc}$ (i.e., the redshift $\left.z \sim 0.085\right)$. This system was analyzed as "System $\mathrm{B}^{\prime \prime}$ in Ref. [38]. We see that the "LISA + B-DECIGO + ET" observation allows us the IMR consistency test at the sub-percent precision both for the GW150914-like, and GW190521like BBH systems. The result of the GW190521-like BBH system is slightly worse than the case of the GW150914-like BBH system because the luminosity distance of GW190521 $\left(D_{L}=5.3 \mathrm{Gpc}\right)$ is much larger than that of GW150914 $\left(D_{L}=0.4 \mathrm{Gpc}\right)[22,107,108]$. Additionally, the GW190521-like BBH system has the central frequency $f_{c} \sim 85 \mathrm{~Hz}$ lower than that of the GW150914-like BBH system $\left(f_{c} \sim 350 \mathrm{~Hz}\right)$, missing the most sensitive frequency of aLIGO and ET $\sim 250 \mathrm{~Hz}$. If a GW190521-like BBH was observed at the same distance as GW150914, the accuracy of its inspiral-ringdown consistency test would be at the level of $\sim O(0.01 \%)$.

\subsection{A Simple Test of the Remnant Compact Object with Quasinormal Modes}

Another simple test of GR is to bracket whether the remnant object should be a BH predicted by GR or not, making use of the parameter estimation errors of QNM frequencies ( $\left.f_{\mathrm{QNM}}=f_{\mathrm{R}}+i f_{\mathrm{I}}\right)$ obtained from the ringdown GW signals [66].

Figure 2 plots the $1 \sigma, 2 \sigma, 3 \sigma, 4 \sigma$, and $5 \sigma$ error contours on the fundamental $(n=0)$, $\ell=2=m$ mode of the QNM frequency in the $\left(f_{\mathrm{R}}, f_{\mathrm{I}}\right)$ plane, in the case of the GW190521like BBH system observed by aLIGO (left) and ET (right). The errors are estimated through the results in Table 3 concerning the parameter estimation error on the ringdown GW signal (after $t_{0}$ and $\phi_{0}$ being marginalized out), and the outermost contour in each panel shows the $5 \sigma$ error. The black lines in each panel depict the Schwarzschild limit of $\left|f_{\mathrm{I}}\right| / f_{\mathrm{R}}$, which may be obtained by setting $\chi_{f}=0$ in Equation (15) with Equation (12):

$$
\frac{\left|f_{\mathrm{I}}\right|}{f_{\mathrm{R}}} \approx 0.236
$$

This is marginally consistent with the exact QNM frequency of the fundamental $(n=0)$, $\ell=2=m$ mode in the Schwarzschild limit. For example, the B.H.P.C. code gives $\left|f_{\mathrm{I}}\right| / f_{\mathrm{R}}=$ $0.23808 \ldots$, and the difference is negligibly small here. The key point of this test is that the top-left side of the black line becomes the prohibited region in GR-i.e., for the $(n=0)$, $\ell=2=m$ mode the QNM frequencies $\left(f_{\mathrm{R}}, f_{\mathrm{I}}\right)$ of any rotating Kerr BHs must sit in the bottom-right side of the black line.

In the aLIGO case, due to the low SNR $(=10.8)$ the parameter estimation errors already go beyond the Schwarzschild limit at the $3 \sigma$ level, and we cannot confirm whether the remnant object is a BH predicted by GR with the $5 \sigma$ level. For such low SNR events, the "coherent mode stacking method" [109] will be useful. On the other hand, thanks to the high SNR (=147) in the ET case, this simple test can necessarily confirm that the remnant object is a GR-predicted BH. Note that the $\left(M_{f}, \chi_{f}\right)$ plane has been discussed as Figure 5 in Ref. [3]. The remnant BH spin is restricted to $0 \leq \chi_{f}<1$ in the analysis. Therefore, the simple test presented in this paper is not applicable.

\section{Summary and Discussion}

This work underlines the multiband observation of the GW190521-like nonprecessing, quasicircular "intermediate-mass" BBH with LISA and B-DECIGO in the low-frequency band, combining it with aLIGO and ET in the high-frequency band. Our first result for the parameter estimation errors is displayed in Tables 2 and 3; the statistical errors of the binary's mass parameters by B-DECIGO observation will be $\sim 10^{-2}$, and the multiband observation with LISA, B-DECIGO, and ET will further improve them to a factor of 2, even allowing the statistical errors of component $\mathrm{BH}$ spins at the accuracy level of $\sim 10^{-1}$. Based on the ringdown analysis, ET will measure the QNM frequency with about $O(0.1) \%$ 
precision. Our second result for the GR tests is presented in Table 4 as well as Figure 2. We showed that the multiband observation of the GW190521-like BBH system by LISA, B-DECIGO, and ET would perform the inspiral-ringdown consistency test at a percent level of precision.

The main point of our analysis is that there is the principal advantage of measuring GW190521-like BBH systems using the full GW spectrum from milli-Hz to deci-Hz bands, and to hecto-Hz band. We expect that our findings will motivate further investigation on prospects for the multiband observation of GW190521-like BBH systems, which are much alike the prototypical GW150914-like BBH systems [22].

Nevertheless, we emphasize that our study was performed with (very) simple methods. Therefore, our results should be only indicative and tentative. In the remainder of this section, we discuss what work remains to be carried out to refine our analysis in the future, so that we can eventually make a strong scientific case for multiband GW astronomy/physics.

\section{Assessment of Prospects}

First, our methodology in Section 2 should be replaced with more modern, sophisticated approaches to the GW data analysis; it will include (but not be limited to), for example, the use of complete IMR waveforms such as EOB approach as well as IMRPhenom models (see, for example, Ref. [110] and the references therein), full-fledged Bayesian posteriorbased techniques (see, for example, Refs. [105,111,112]), and more 'realistic' noise and waveform models that account for the sky-location of sources as well as orbital configurations of B-DECIGO [11] and LISA [8].

Second, our target GW190521-like BBH system was restricted to the nonprecessing, spinning, quasicircular configuration; although there is large uncertainty, the spin precession of GW190521 is estimated as nonzero [3,4]. Future studies are therefore needed to concern both the spin precession and orbital eccentricity of the BBH system: we will briefly discuss the eccentric, non-spinning BBH system in Appendix A. In general, the BH's spins in the precessing binary have not only their magnitudes but also orientations, which can be described by, for example, the effective inspiral spin parameter $\chi_{\text {eff }}$ (related to the components aligned with the orbital angular momentum) and the precession spin parameter $\chi_{\mathrm{p}}$ (related to the components in the orbital plane for the inspiral GW waveform). Adding the orbital eccentricity to the (precessing) BBH system will be fully generic, and the waveform modeling becomes (much) more complicated. Despite that challenge, there is a considerable development of the analysis on fully generic binary systems [113].

Related to the point mentioned above, it should be noted that we have ignored subdominant $(\ell \neq 2,|m| \neq 2)$ harmonics in our GW waveform model. They are more notable in the observed signal when system's mass ratio becomes smaller (such as the analysis of GW190814 [114] with the mass ratio $q=0.112_{-0.009}^{+0.008}$ ). With the subdominant harmonics, one can access the source orientation to reduce uncertainty in the distance estimation [115].

Third, we should note that there are two main hurdles to analyze the ringdown GW signals; the low SNR with aLIGO and the starting time of the ringdown phase that is a priori unknown to the whole observed GW signal (see, for example, Refs. [116,117] for discussions on the starting time). Our simple analysis is carried out with the single-mode waveform model (11) as the template to analyze the ringdown phase, assuming the starting time of the ringdown phase to be $t=t_{0}$, which corresponds to $f=f_{\mathrm{R}}$-i.e., just after the end of the merger phase. While this choice does not affect our results of parameter estimation errors, in practice the best fit values (that should be obtained rather than assumed in the context of the full-parameter estimation against the raw GW data) can be biased if one assumes the earlier starting time in the analysis (see, for example, Figure 5 in Ref. [88]). Although one may delay the starting time to avoid the bias in the parameter estimation, the SNR becomes much lower than the expected SNR with the assumption of $t=t_{0}$. 
These obstacles will be overcome if one includes higher overtones $(n>0)$ into the ringdown GW analysis [85], for which a much larger SNR than the single-mode analysis will be expected. Indeed, a superposition of overtones $(n>0)$ in a single harmonic mode will be observed [85,118-120] in the high SNR events (with ET). Another step-functional improvement of the ringdown GW analysis will be offered by using completely different signal analysis methods than the traditional matched filtering analysis, which may not be always optimal for the ringdown GW signal. There is ongoing work to assess the improvement due to new techniques for the ringdown GW signal analysis (such as HilbertHuang transformation, autoregressive modeling, and neural networks) [121,122].

Fourth, there are other GR tests that were not covered in Section 4 but that can be greatly improved using the multiband observation of GW195021-like BBH systems. Reference [91] performs various tests of GR with the BBH events in GWTC-2 (see also the reviews by Carson and Yagi [123] about the current and future test with GWs), including (i) the IMR consistency test between the inspiral and postinspiral phases divided at some cutoff frequency; (ii) constraining deviations from GR with parametric deformations to a predicted GR waveform model, (iii) "BH spectroscopy" [124,125] with ringdown GWs which contains two (or possibly more) QNMs [126,127], and so on.

The test (i) is similar to our inspiral-ringdown consistency test directly using the information of the merger phase, and makes the most of the GW waveform. It has been pointed out that the values of this test with GW150914-like BBH systems will be maximized in the multiband observation $[28,29,128]$. Our result suggests that the same will be true for the GW190521-like "intermediate-mass" BBH systems, too. Similarly, Gupta et al. [31,32] have showed that the multiband observations of stellar- and intermediate-mass BBHs with LISA and 3G detectors will be only workable way to carry out the most general version of test (ii). Adding B-DECIGO (or any other planned GW detectors) in the deci-Hz band to the multiband analysis, we expect the precision of this test will be unprecedented.

Based on the generalized likelihood ratio test [129], the test (iii) is performed (see, for example, Section 9.5 in Ref. [130] and references therein, and also Ref. [131] for the future $\mathrm{O} 5 \mathrm{era}$ ). This test with aLIGO and Advanced Virgo alone is quite challenging to have any conclusive result, simply because of the too low SNRs and larger parameter estimation errors in the ringdown phase. In the $3 \mathrm{G}$ era, two (or possibly more) QNMs will be measurable [126,127]. It is also helpful to use the multiband observation in order to optimize ground-based detectors via the forewarnings from the low-frequency, LISA band [132]. In either cases, the multiband observation with B-DECIGO and ET will give the additional advantage of being able to perform the best test of Kerr hypothesis of remnant $\mathrm{BHs}$ via $\mathrm{BH}$ spectroscopy.

Author Contributions: The authors contribute equally to this paper. All authors have read and agreed to the published version of the manuscript.

Funding: H.N. acknowledges support from JSPS KAKENHI Grant No. JP16K05347. S.I. acknowledges support from STFC through Grant No. ST/R00045X/1. S.I. also thanks to networking support by the GWverse COST Action CA16104, "Black holes, gravitational waves and fundamental physics". N.S. and H.N. acknowledge support from JSPS KAKENHI Grant No. JP17H06358.

Informed Consent Statement: Not applicable.

Data Availability Statement: The data presented in this study are available on request from the corresponding author.

Acknowledgments: We would like to thank Carlos O. Lousto, James Healy. and Leor Barack for useful discussion. We also express our sincere gratitude to anonymous referees, who gave us valuable comments and kindly pointed out many typos in a previous version of this manuscript. All the analytical and numerical calculations in this paper were performed with Maple and Black Hole Perturbation Club (B.H.P.C.) codes [75].

Conflicts of Interest: The authors declare no conflict of interest. 


\section{Appendix A. Signal-to-Noise Ratio of GW190521-Like Eccentric BBH Systems}

Throughout the bulk of this paper, we have looked at the GW190521-like BBH system under the assumption of a quasicircular $\mathrm{BBH}$ merger. While the quasicircular evolution of GW190521 is totally consistent with the LIGO/Virgo observation [3,4], due to the lack of the inspiral GW signal long enough, it appears that alternative scenarios, for example, GW190521 as an eccentric BBH merger also become relevant. Indeed, Gayathri et al. [44] demonstrated that observed GW190521 data could be explained as an equal-mass, highly eccentric $(e=0.7) \mathrm{BBH}$ system (the orbital eccentricity $(e=0.7)$ is provided as initial data for NR simulations at a frequency of $10 \mathrm{~Hz}$ for a system with the total mass of $50 \mathrm{M}_{\odot}$ that is the orbital separation $\sim 24.5 m_{t}$ for BBHs with the total mass $m_{t}$ [133] and note that the NR waveforms are scaled by the total mass $m_{t}$ ), and the estimated source parameters are quite different from those derived from the quasicircular BBH scenario (recall Table 1): the primary mass $m_{1}^{\mathrm{r}}=102_{-11}^{+7} M_{\odot}$, the secondary mass $m_{2}^{\mathrm{r}}=102_{-11}^{+7} M_{\odot}$, and the total mass $m_{t}^{\mathrm{r}}=204_{-33}^{+14} M_{\odot}$ in the source's rest frame, the mass ratio $q=1$, the luminosity distance $D_{L}=1.84_{-0.054}^{+1.07} \mathrm{Gpc}$ and the redshift $z=0.35_{-0.09}^{+0.16}$ (see Ref. [44] for the spin parameters). The possibility of GW190521 with nonvanishing eccentricity is also pointed out by Refs. [134,135].

Like the quasicircular case, the multiband observation of eccentric GW190521-like BBH systems will once again help in distinguishing these two scenarios. Assuming a quadrupole GW generation from a Newtonian Kepler orbit [136,137], the typical coalescing time $t_{c}^{\text {ecc }}$ and the characteristic strain amplitude $h_{c, n}^{\mathrm{ecc}}$ of the $n$-th harmonic are (see, for example, Section 4.1 of Maggiore's text [138] as well as Ref. [139]):

$$
t_{c}^{\mathrm{ecc}} \sim t_{c}^{\mathrm{circ}}\left(1-e_{0}^{2}\right)^{7 / 2}, \quad h_{c}^{\mathrm{ecc}} \sim h_{c}^{\mathrm{circ}} g(n, e),
$$

where $t_{c}^{\text {circ }}$ and $h_{c}^{\text {circ }}$ are corresponding circular-inspiral results given in Equations (1) and (3), respectively, and the function $g(n, e)$ will be defined momentarily. We see that the early inspiral phase of the eccentric binary is well within the B-DECIGO and LISA bands, too. Specifically, Holgado et al. [140] (see also the works by Amaro-Saoane [42,139,141]) pointed out that having deci-Hz GW observatories such as B-DECIGO, MAGIS [14] and TianGO [15] will be a key element to observe the eccentric inspiral GW signals in multiband networks because the GW signals may skip the LISA band entirely; $h_{c, n}^{\text {ecc }}$ can be suppressed by a function of $g(n, e)$ significantly, depending on its eccentricity in the LISA band.

To better understand the visibility of eccentric BBH systems with aLIGO, ET, BDECIGO, and LISA, let us estimate the SNR of non-spinning, eccentric BBH inspirals accumulated in each band; see also Ref. [142] for a recent review about waveform families for the eccentric binary systems. We also see various active works on eccentric waveform approximants [143-146]. The squared SNR averaged over the all-sky positions and binary orientations may be written as $[23,53,81,147]$ :

$$
\rho_{\text {ave }}^{2} \approx \frac{1}{5} \sum_{n} \int_{f_{\text {in }}}^{f_{\text {end }}} \frac{h_{c, n}^{2}}{f_{n} S(f)} d\left(\ln f_{n}\right),
$$

where $S(f)$ is the noise PSD for a given GW detector, and

$$
f_{n}=n f_{\text {orb }}=n f_{\text {orb }}^{\mathrm{r}}(1+z)^{-1},
$$

is the frequency of the harmonic in the observer frame, defined by the source's rest-frame frequency $f_{\text {orb }}^{\mathrm{r}}$ of the Kepler orbit with the redshift $z$. The expression for the average SNR (A2) recovers Equation (17) (of the inspiral part) in the circular orbit limit, $e \rightarrow 0$ (note that the harmonics are restricted to only $n=2$ in the circular limit because one has $\left.\lim _{e \rightarrow 0} g(n, e)=\delta_{2, n}\right)$. The dimensionless characteristic strain $h_{c, n}$ of the $n$-th harmonic is [147]:

$$
h_{c, n} \equiv \frac{1+z}{\pi D_{L}} \sqrt{2 \frac{d E_{n}^{\mathrm{r}}}{d f_{n}^{\mathrm{r}}}}
$$


where $D_{L}$ is the luminosity distance. Assuming the quadrupole formula applied to the Kepler orbit (with the chirp mass $\mathcal{M}$ in the observer frame) [136,137],

$$
\frac{d E_{n}^{\mathrm{r}}}{d f_{n}^{\mathrm{r}}} \equiv \frac{\pi}{3} \frac{\mathcal{M}^{5 / 3}}{(1+z)^{2}} \frac{g(n, e)}{F(e)}\left(\frac{2}{n}\right)^{2 / 3}\left(\pi f_{n}\right)^{-1 / 3},
$$

is the emitted GW energy per unit frequency $f_{n}^{\mathrm{r}}$ at the $n$-th harmonic measured in the source's rest frame, and we define:

$$
\begin{gathered}
g(n, e) \equiv \frac{n^{4}}{32}\left\{\left[J_{n-2}(n e)-2 e J_{n-1}(n e)+\frac{2}{n} J_{n}(n e)+2 e J_{n+1}(n e)-J_{n+2}(n e)\right]^{2}\right. \\
\left.+\left(1-e^{2}\right)\left[J_{n-2}(n e)-2 J_{n}(n e)+J_{n+2}(n e)\right]^{2}+\frac{4}{3 n^{2}} J_{n}^{2}(n e)\right\} \\
F(e) \equiv \frac{1+\frac{73}{24} e^{2}+\frac{37}{96} e^{4}}{\left(1-e^{2}\right)^{7 / 2}}\left(=\sum_{n=1}^{\infty} g(n, e)\right)
\end{gathered}
$$

with the Bessel functions of the first kind $J_{n}(x)$ ( $n$ : integer); see, for example, Ref. [148] for the derivation of Equation (A5).

For an inspiraling eccentric BBH, the evaluation of SNR through Equation (A2) requires the knowledge of the slowly evolving orbital eccentricity $e$ and the frequency $f_{n}$ in time, under the gravitational radiation losses. Again, in the quadrupole formalism, this is given by [136,137]:

$$
\frac{f_{\text {orb }}}{f_{\text {orb }, 0}}=\left[\frac{1-e_{0}^{2}}{1-e^{2}}\left(\frac{e}{e_{0}}\right)^{12 / 19}\left(\frac{1+\frac{121}{304} e^{2}}{1+\frac{121}{304} e_{0}^{2}}\right)^{870 / 2299}\right]^{-3 / 2},
$$

with reference eccentricity $e_{0}$ and orbital frequency $f_{\text {orb }, 0}$ (in the observer frame). One can set these constants by the values at the last stable orbit (LSO) of the eccentric geodesic (of a test particle) in the Schwarzschild geometry [149]: namely, $e_{0}=e_{\mathrm{LSO}}$ and:

$$
\begin{aligned}
f_{\text {orb }, 0}=f_{\mathrm{LSO}} & =\frac{1}{2 \pi(1+z) m_{t}^{\mathrm{r}}}\left(\frac{1-e_{\mathrm{LSO}}^{2}}{6+2 e_{\mathrm{LSO}}}\right)^{3 / 2} \\
& \sim 8.0(1+z)^{-1}\left(\frac{m_{t}^{\mathrm{r}}}{204 M_{\odot}}\right)^{-1}\left(\frac{1-e_{\mathrm{LSO}}^{2}}{6+2 e_{\mathrm{LSO}}}\right)^{3 / 2} \mathrm{~Hz},
\end{aligned}
$$

with the total mass $m_{t}^{\mathrm{r}}$ in the source's rest frame. The frequency evolution (A8) is therefore completely determined with a given single parameter $e_{\mathrm{LSO}}$.

We compute the averaged SNR $\rho_{\text {ave }}$ given in Equation (A2) for the GW190521-like, non-spinning, eccentric BBHs with the source-frame masses $\left(m_{1}^{\mathrm{r}}, m_{2}^{\mathrm{r}}\right)=\left(102 M_{\odot}, 102 M_{\odot}\right)$ and the luminosity distance $D_{L}=1.9 \mathrm{Gpc}$ (i.e., the redshift $z \sim 0.35$ ), which mimics the eccentric BBH merger obtained by the NR simulations in Ref. [44]. We assume the five year observation prior to the final merger determined by Equations (A1) and (A9), and apply the same setup described in Section 3.1 to each $n$-th harmonic of GW strains. Because the frequency evolution (A8) is expressed in term of eccentricity, in practice, we change the integration variable of Equation (A2) from $f_{n}$ to $e$ for the computational efficiency, making use of $d f_{n}=n\left|d f_{\text {orb }} / d e\right| d e$ with (see, for example, Ref. [150]):

$$
\frac{d f_{\text {orb }}}{d e}=-\frac{18}{19} \frac{f_{\text {orb }}}{e} \frac{F(e)}{\left(1-e^{2}\right)^{9 / 2}\left(1+\frac{121}{304} e^{2}\right)} .
$$

Additionally, the infinite summation over the harmonics $n$ in Equation (A2) is truncated at some finite value of $n_{\max }$; we chose $n_{\max }=O\left(10^{4}\right)$ so that the resultant SNRs are 
guaranteed to have at least 3 significant digits (a good analytical estimator of $n_{\max }$ can be found in, for examples, Refs. [151,152]).

In Table A1, we summarize the averaged SNRs $\rho_{\text {ave }}$ of the GW190521-like, nonspinning, eccentric BBH accumulated in each GW band, for sample values of final eccentricities at the last stable orbit $e_{\mathrm{LSO}}=\left\{10^{-6}, 10^{-3}, 0.1,0.4,0.6\right\}$. For references, the 'initial' eccentricities at $f_{\text {in }}$ for each band are also listed. It is approximated by solving Equation (A8) for $e$ at the detector's initial GW frequency of the second harmonics $f_{\text {orb }}=f_{\text {in }} / 2$. We found three main results: (i) B-DECIGO and ET always accumulate a total SNR greater than at least 10 , independent of the value of the final eccentricity $e_{\mathrm{LSO}}$; (ii) LISA has the detectable SNR only when $e_{\mathrm{LSO}}<10^{-3}$. That is, the inspiral GW signal from the GW190521-like $\mathrm{BBH}$ that has the high-eccentricity in the aLIGO band would entirely skip the LISA band; (iii) the SNR with B-DECIGO becomes bigger when $e_{\mathrm{LSO}}$ becomes smaller, while the SNR with ET and aLIGO shows the opposite behavior. Therefore, the multiband observation could provide much louder SNR than the single-band SNR across the full range of $e_{\mathrm{LSO}}$. Using Equation (21) with the result in Table A1, we find that the multiband SNR with B-DECIGO and ET are $\sim 180$ for $e_{\mathrm{LSO}}=\left\{10^{-6}, 10^{-3}\right\}, \sim 140$ for $e_{\mathrm{LSO}}=\{0.4,0.6\}$ and $\sim 100$ for $e_{\mathrm{LSO}}=0.1$.

Table A1. Averaged SNRs $\rho_{\text {ave }}$ of the GW190521-like, non-spinning, eccentric BBHs accumulated in each band five years prior to coalescence, for given values of final eccentricity $e=e_{\mathrm{LSO}}$. We assume the source-frame component masses $\left(m_{1}^{\mathrm{r}}, m_{2}^{\mathrm{r}}\right)=\left(102 M_{\odot}, 102 M_{\odot}\right)$, and the luminosity distance $D_{L}=1.9 \mathrm{Gpc}$ (i.e., the redshift $z \sim 0.35$ ) [44]. The values in parentheses indicate the 'initial' eccentricity estimated from the initial GW frequency ( $f_{\text {in }}$ defined in Section 3$)$ of the second harmonic mode in each detectors. Note that aLIGO's 'initial' eccentricity when $e_{\mathrm{LSO}}=0.6$ is not displayed because the second harmonic mode is not detectable in this case.

\begin{tabular}{cccccc}
\hline \multicolumn{6}{c}{ SNR and Eccentricity at $f_{\text {in }}$} \\
\hline & $\boldsymbol{e}_{\text {LSO }}=\mathbf{1 0}^{-\mathbf{6}}$ & $\boldsymbol{e}_{\text {LSO }}=\mathbf{1 0}^{-\mathbf{3}}$ & $\boldsymbol{e}_{\text {LSO }}=\mathbf{0 . 1}$ & $\boldsymbol{e}_{\text {LSO }}=\mathbf{0 . 4}$ & $\boldsymbol{e}_{\text {LSO }}=\mathbf{0 . 6}$ \\
\hline \multirow{2}{*}{ aLIGO } & 4.95 & 4.95 & 5.55 & $1.07 \times 10^{1}$ & $1.11 \times 10^{1}$ \\
& $\left(1.64 \times 10^{-6}\right)$ & $\left(1.64 \times 10^{-3}\right)$ & $(0.150)$ & $(0.405)$ & $(\cdots)$ \\
\hline ET & $7.64 \times 10^{1}$ & $7.63 \times 10^{1}$ & $8.73 \times 10^{1}$ & $1.42 \times 10^{2}$ & $1.42 \times 10^{2}$ \\
& $\left(8.98 \times 10^{-6}\right)$ & $\left(8.97 \times 10^{-3}\right)$ & $(0.505)$ & $(0.746)$ & $(0.793)$ \\
\hline B-DECIGO & $1.67 \times 10^{2}$ & $1.70 \times 10^{2}$ & $5.08 \times 10^{1}$ & $1.85 \times 10^{1}$ & $1.20 \times 10^{1}$ \\
& $\left(2.41 \times 10^{-3}\right)$ & $(0.709)$ & $(0.982)$ & $(0.992)$ & $(0.993)$ \\
\hline LISA & 7.60 & 2.05 & $<1.00$ & $<1.00$ & $<1.00$ \\
& $\left(3.86 \times 10^{-3}\right)$ & $(0.985)$ & $(>0.999)$ & $(>0.999)$ & $(>0.999)$ \\
\hline
\end{tabular}

Finally, although the parameter estimation is not the main focus here, we briefly discuss the potential accuracy of the eccentricity measurement. In the small-eccentricity and high-SNR limit, the orbital eccentricity may be measured within the fractional error [153] (see also Ref. [154]):

$$
\delta \hat{e}_{0} \sim 5 \times 10^{-5} \frac{(1+z)^{5 / 3}}{\sqrt{2+3 \alpha}}\left(\frac{\mathcal{M}^{\mathrm{r}}}{65.1 M_{\odot}}\right)^{5 / 3}\left(\frac{f_{0}}{0.1 \mathrm{~Hz}}\right)^{5 / 3}\left(\frac{e_{0}}{0.1}\right)^{-1},
$$

from Equation (2) in Ref. [153] by using a rough approximation with the quasicircular amplitude and the eccentric phase, where $\delta \hat{e}_{0}$ denotes the parameter estimation error of $e_{0}$ (at the GW frequency $f_{0}$ for $n=2$ ) normalized by the SNR. Here, the power $\alpha$ is due to the approximation of the noise PSD by the power law, $S_{n} \sim f^{2 \alpha}$ (assuming $\alpha>-2 / 3$ ), and B-DECIGO may have $\alpha=1$, for example. This estimator implies that B-DECIGO would be able to precisely measure the eccentricity of GW190521-like BBH systems. Because we find that the 'B-DECIGO + ET' combination always provides the multiband SNRs larger than $\sim 100$ independent of the value of $e_{\mathrm{LSO}}$, one might expect that this combination best 
observes GW190521-like BBHs over the full range of eccentricity, helping to understand the population properties of BBH mergers [155]. We will explore this possibility in future work.

\section{Appendix B. Some More Noise Power Spectral Densities of (Next-Generation) GW Detectors}

In this appendix, we summarize some (fitting) curves of the noise PSD for both ground and space-based, current and future GW detectors. These sensitivity curves are not used in the bulk of this paper, but it will serve as a convenient all-in-one-place summary with our notation; these curves with $f$ in unit of $\mathrm{Hz}$ are shown in Figure 4 of Ref. [156] (except DECIGO and TianQin).

- "LIGO O3a-Livingston" rough fitting curve (during the first half of LIGO/Virgo third observing run by using Ref. [157]):

$$
\begin{aligned}
S_{n}^{\mathrm{O} 3 \mathrm{a}-\mathrm{L}}=( & 2.13068 \times 10^{-12} f^{-7.938724592}+4.0 \times 10^{-22} f^{-1.0} \\
& \left.+3.0 \times 10^{-24}+1.74546 \times 10^{-27} f^{1.178746922}\right)^{2} \mathrm{~Hz}^{-1} .
\end{aligned}
$$

- "LIGO O5" rough fitting curve (will be in the fifth observing run by using Ref. [157]):

$$
\begin{aligned}
S_{n}^{\mathrm{O} 5}= & \left(480985000.0 f^{-30.28419138}+6.63263 \times 10^{-20} f^{-3.122716032}\right. \\
& \left.+6.15101 \times 10^{-21} f^{-2.089976737}+1.32853 \times 10^{-27} f^{1.059219544}\right)^{2} \mathrm{~Hz}^{-1} .
\end{aligned}
$$

- "ET-B" (another sensitivity curve for ET in Ref. [158] other than Equation (5); see also, for examples, Ref. [159] and ET sensitivity page [160]):

$$
\begin{aligned}
S_{n}^{\mathrm{ET}-\mathrm{B}}=1.0 \times 10^{-50}( & 45540.5 f^{-15.64}+6804.96 f^{-2.145} \\
& \left.+3.05853 f^{-0.12}+0.00258062 f^{1.1}\right)^{2} \mathrm{~Hz}^{-1} .
\end{aligned}
$$

- "CE2" rough fitting curve (for Cosmic Explorer presented in Ref. [21]):

$$
S_{n}^{\mathrm{CE} 2}=\left(1.74408 \times 10^{-16} f^{-8.908164528}+2.0 \times 10^{-25}+8.23008 \times 10^{-32} f^{2.095903274}\right)^{2} \mathrm{~Hz}^{-1} .
$$

- "DECIGO" (the noise PSD of the L-shaped configuration [161]):

$$
\begin{aligned}
S_{n}^{\text {DECIGO }}=\{ & 7.05 \times 10^{-48}\left[1+\left(\frac{f}{f_{p}}\right)^{2}\right]+4.8 \times 10^{-51} f^{-4}\left[1+\left(\frac{f}{f_{p}}\right)^{2}\right]^{-1} \\
+ & \left.5.53 \times 10^{-52} f^{-4}\right\} \mathrm{Hz}^{-1} .
\end{aligned}
$$

with $f_{p} \equiv 7.36$. Note that this expression accounted for the factor of $(\sqrt{3} / 2)^{-2}$ due to DECIGO having arms that make an angle of $60^{\circ}$.

- "TianQin" $[13,162]$ (the sky averaged noise PSD; the expression below is quoted from Equations (9) and (10) of Ref. [163]): 


$$
\begin{aligned}
S_{n}^{\text {TianQin }}=3.0 \times 10^{-51}( & 0.009505539123 f^{-5}+95.05539123 f^{-4}+0.07550033531 f^{-3} \\
& +755.0033531 f^{-2}+3.703703703 \times 10^{10} \\
& \left.+2.941767614 \times 10^{11} f^{2}\right) \mathrm{Hz}^{-1}
\end{aligned}
$$

\section{References}

1. Abbott, B.P.; Abbott, R.; Abbott, T.D.; Abraham, S.; Acernese, F.; Ackley, K.; Adams, C.; Adhikari, R.X.; Adya, V.B.; Affeldt, C.; et al. GWTC-1: A Gravitational-Wave Transient Catalog of Compact Binary Mergers Observed by LIGO and Virgo during the First and Second Observing Runs. Phys. Rev. X 2019, 9, 031040. [CrossRef]

2. Abbott, R.; Abbott, T.D.; Abraham, S.; Acernese, F.; Ackley, K.; Adams, A.; Adams, C.; Adhikari, R.X.; Adya, V.B.; Affeldt, C.; et al. GWTC-2: Compact Binary Coalescences Observed by LIGO and Virgo During the First Half of the Third Observing Run. arXiv 2010, arXiv:2010.14527.

3. Abbott, R.; Abbott, T.D.; Abraham, S.; Acernese, F.; Ackley, K.; Adams, C.; Adhikari, R.X.; Adya, V.B.; Affeldt, C.; Agathos, M.; et al GW190521: A Binary Black Hole Merger with a Total Mass of 150M $\odot$. Phys. Rev. Lett. 2020, 125, 101102. [CrossRef]

4. Abbott, R.; Abbott, T.D.; Abraham, S.; Acernese, F.; Ackley, K.; Adams, C.; Adhikari, R.X.; Adya, V.B.; Affeldt, C.; Agathos, M.; et al. Properties and Astrophysical Implications of the $150 \mathrm{M}_{\odot}$ Binary Black Hole Merger GW190521. Astrophys. J. Lett. 2020, 900, L13. [CrossRef]

5. Ade, P.A.R.; Aghanim, N.; Arnaud, M.; Ashdown, M.; Aumont, J.; Baccigalupi1, C.; Banday, A.J.; Barreiro, R.B.; Bartlett, J.G.; Bartolo, N.; et al. Planck 2015 results. XIII. Cosmological parameters. Astron. Astrophys. 2016, 594, A13. [CrossRef]

6. Varma, V.; Field, S.E.; Scheel, M.A.; Blackman, J.; Gerosa, D.; Stein, L.C.; Kidder, L.E.; Pfeiffer, H.P. Surrogate models for precessing binary black hole simulations with unequal masses. Phys. Rev. Res. 2019, 1, 033015. [CrossRef]

7. Nitz, A.H.; Capano, C.D. GW190521 may be an intermediate mass ratio inspiral. arXiv 2010, arXiv:2010.12558.

8. Amaro-Seoane, P.; Audley, H.; Babak, S.; Baker, J.; Barausse, E.; Bender, P.; Berti, E.; Binetruy, P.; Born, M.; Bortoluzzi, D.; et al. Laser Interferometer Space Antenna. arXiv 2017, arXiv:1702.00786.

9. Nakamura, T.; Ando, M.; Kinugawa, T.; Nakano, H.; Eda, K.; Sato, S.; Musha, M.; Akutsu, T.; Tanaka, T.; Seto, N.; et al. Pre-DECIGO can get the smoking gun to decide the astrophysical or cosmological origin of GW150914-like binary black holes. Prog. Theor. Exp. Phys. 2016, 2016, 093E01. [CrossRef]

10. Seto, N.; Kawamura, S.; Nakamura, T. Possibility of direct measurement of the acceleration of the universe using $0.1-\mathrm{Hz}$ band laser interferometer gravitational wave antenna in space. Phys. Rev. Lett. 2001, 87, 221103. [CrossRef] [PubMed]

11. Kawamura, S.; Ando, M.; Seto, N.; Sato, S.; Musha, M.; Kawano, I.; Yokoyama, J.; Tanaka, T.; Ioka, K.; Akutsu, T.; et al. Current status of space gravitational wave antenna DECIGO and B-DECIGO. arXiv 2020, arXiv:2006.13545.

12. Ruan, W.H.; Guo, Z.K.; Cai, R.G.; Zhang, Y.Z. Taiji program: Gravitational-wave sources. Int. J. Mod. Phys. A 2020, $35,2050075$. [CrossRef]

13. Luo, J.; Chen, L.-S.; Duan, H.-Z.; Gong, Y.-G.; Hu, S.; Ji, J.; Liu, Q.; Mei, J.; Milyukov, V.; Sazhin, M. TianQin: A space-borne gravitational wave detector. Class. Quant. Grav. 2016, 33, 035010. [CrossRef]

14. Graham, P.W.; Jung, S. Localizing Gravitational Wave Sources with Single-Baseline Atom Interferometers. Phys. Rev. D 2018, 97, 024052. [CrossRef]

15. Kuns, K.A.; Yu, H.; Chen, Y.; Adhikari, R.X. Astrophysics and cosmology with a decihertz gravitational-wave detector: TianGO. Phys. Rev. D 2020, 102, 043001. [CrossRef]

16. Ni, W.T. Gravitational wave detection in space. Int. J. Mod. Phys. D 2016, 25, 1630001. [CrossRef]

17. Ni, W.T. Mid-frequency gravitational wave detection and sources. Int. J. Mod. Phys. D 2020, 29, 1902005. [CrossRef]

18. Akutsu, T.; Ando, M.; Arai, K.; Arai, Y.; Araki, S.; Araya, A.; Aritomi, N.; Aso, Y.; Bae, S.-W.; Bae, Y.-B.; et al. Overview of KAGRA: Detector design and construction history. arXiv 2020, arXiv:2005.05574.

19. Hild, S.; Chelkowski, S.; Freise, A.; Franc, J.; Morgado, N.; Flaminio, R.; DeSalvo, R. A Xylophone Configuration for a third Generation Gravitational Wave Detector. Class. Quant. Grav. 2010, 27, 015003. [CrossRef]

20. Punturo, M.; Abernathy, M.; Acernese, F.; Allen, B.; Andersson, N.; Arun, K.; Barone, F.; Barr, B.; Barsuglia, M.; Beker, M.; et al. The Einstein Telescope: A third-generation gravitational wave observatory. Class. Quant. Grav. 2010, 27, 194002. [CrossRef]

21. Reitze, D.; Adhikari, R.X.; Ballmer, S.; Barish, B.; Barsotti, L.; Billingsley, G.; Brown, D.A.; Chen, Y.; Coyne, D.; Eisenstein, R.; et al. Cosmic Explorer: The U.S. Contribution to Gravitational-Wave Astronomy beyond LIGO. Bull. Am. Astron. Soc. $2019,51,035$.

22. Abbott, B.P.; Abbott, R.; Abbott, T.D.; Abernathy, M.R.; Acernese, F.; Ackley, K.; Adams, C.; Adams, T.; Addesso, P.; Adhikari, R.X.; et al. Observation of Gravitational Waves from a Binary Black Hole Merger. Phys. Rev. Lett. 2016, 116, 061102. [CrossRef]

23. Sesana, A. Prospects for Multiband Gravitational-Wave Astronomy after GW150914. Phys. Rev. Lett. 2016, 116, 231102. [CrossRef]

24. Cutler, C.; Berti, E.; Jani, K.; Kovetz, E.D.; Randall, L.; Vitale, S.; Wong, K.W.K.; Holley-Bockelmann, K.; Larson, S.L.; Littenberg, T.; et al. What we can learn from multi-band observations of black hole binaries. arXiv 2019, arXiv:1903.04069.

25. Vitale, S. Multiband Gravitational-Wave Astronomy: Parameter Estimation and Tests of General Relativity with Space- and Ground-Based Detectors. Phys. Rev. Lett. 2016, 117, 051102. [CrossRef] [PubMed] 
26. Jani, K.; Shoemaker, D.; Cutler, C. Detectability of Intermediate-Mass Black Holes in Multiband Gravitational Wave Astronomy. Nat. Astron. 2019, 4, 260-265. [CrossRef]

27. Barausse, E.; Yunes, N.; Chamberlain, K. Theory-Agnostic Constraints on Black-Hole Dipole Radiation with Multiband Gravitational-Wave Astrophysics. Phys. Rev. Lett. 2016, 116, 241104. [CrossRef]

28. Carson, Z.; Yagi, K. Multi-band gravitational wave tests of general relativity. Class. Quant. Grav. 2020, 37, 02LT01. [CrossRef]

29. Carson, Z.; Yagi, K. Parametrized and inspiral-merger-ringdown consistency tests of gravity with multiband gravitational wave observations. Phys. Rev. D 2020, 101, 044047. [CrossRef]

30. Toubiana, A.; Marsat, S.; Babak, S.; Barausse, E.; Baker, J. Tests of general relativity with stellar-mass black hole binaries observed by LISA. Phys. Rev. D 2020, 101, 104038. [CrossRef]

31. Gupta, A.; Datta, S.; Kastha, S.; Borhanian, S.; Arun, K.G.; Sathyaprakash, B.S. Multiparameter tests of general relativity using multiband gravitational-wave observations. Phys. Rev. Lett. 2020, 125, 201101. [CrossRef]

32. Datta, S.; Gupta, A.; Kastha, S.; Arun, K.G.; Sathyaprakash, B.S. Tests of general relativity using multiband observations of intermediate mass binary black hole mergers. Phys. Rev. D 2021, 103, 024036. [CrossRef]

33. Gerosa, D.; Ma, S.; Wong, K.W.K.; Berti, E.; O'Shaughnessy, R.; Chen, Y.; Belczynski, K. Multiband gravitational-wave event rates and stellar physics. Phys. Rev. D 2019, 99, 103004. [CrossRef]

34. Ng, K.K.Y.; Isi, M.; Haster, C.J.; Vitale, S. Multiband gravitational-wave searches for ultralight bosons. Phys. Rev. D 2020, 102, 083020. [CrossRef]

35. Toubiana, A.; Sberna, L.; Caputo, A.; Cusin, G.; Marsat, S.; Jani, K.; Babak, S.; Barausse, E.; Caprini, C.; Pani, P.; et al. Detectable environmental effects in GW190521-like black-hole binaries with LISA. arXiv 2020, arXiv:2010.06056.

36. Ewing, B.; Sachdev, S.; Borhanian, S.; Sathyaprakash, B.S. Archival searches for stellar-mass binary black holes in LISA. arXiv 2020, arXiv:2011.03036.

37. Nair, R.; Jhingan, S.; Tanaka, T. Synergy between ground and space based gravitational wave detectors for estimation of binary coalescence parameters. Prog. Theor. Exp. Phys. 2016, 2016, 053E01. [CrossRef]

38. Isoyama, S.; Nakano, H.; Nakamura, T. Multiband Gravitational-Wave Astronomy: Observing binary inspirals with a decihertz detector, B-DECIGO. Prog. Theor. Exp. Phys. 2018, 2018, 073E01. [CrossRef]

39. Nair, R.; Tanaka, T. Synergy between ground and space based gravitational wave detectors. Part II: Localisation. J. Cosmol. Astropart. Phys. 2018, 8, 033. [CrossRef]

40. Grimm, S.; Harms, J. Multiband gravitational-wave parameter estimation: A study of future detectors. Phys. Rev. D 2020, 102, 022007. [CrossRef]

41. Liu, C.; Shao, L.; Zhao, J.; Gao, Y. Multiband Observation of LIGO/Virgo Binary Black Hole Mergers in the Gravitational-wave Transient Catalog GWTC-1. Mon. Not. R. Astron. Soc. 2020, 496, 182-196. [CrossRef]

42. Amaro-Seoane, P.; Santamaria, L. Detection of IMBHs with ground-based gravitational wave observatories: A biography of a binary of black holes, from birth to death. Astrophys. J. 2010, 722, 1197-1206. [CrossRef]

43. Yagi, K. Gravitational wave observations of galactic intermediate-mass black hole binaries with DECIGO Path Finder. Class. Quant. Grav. 2012, 29, 075005. [CrossRef]

44. Gayathri, V.; Healy, J.; Lange, J.; O’Brien, B.; Szczepanczyk, M.; Bartos, I.; Campanelli, M.; Klimenko, S.; Lousto, C.; O'Shaughnessy, R. GW190521 as a Highly Eccentric Black Hole Merger. arXiv 2020, arXiv:2009.05461.

45. Ajith, P. Addressing the spin question in gravitational-wave searches: Waveform templates for inspiralling compact binaries with nonprecessing spins. Phys. Rev. D 2011, 84, 084037. [CrossRef]

46. Sathyaprakash, B.S.; Schutz, B.F. Physics, Astrophysics and Cosmology with Gravitational Waves. Living Rev. Rel. 2009, 12, 2. [CrossRef]

47. Robson, T.; Cornish, N.J.; Liu, C. The construction and use of LISA sensitivity curves. Class. Quant. Grav. 2019, 36, 105011. [CrossRef]

48. Nagar, A.; Bernuzzi, S.; Pozzo, W.D.; Riemenschneider, G.; Akcay, S.; Carullo, G.; Fleig, P.; Babak, S.; Tsang, K.W.; Colleoni, M.; et al. Time-domain effective-one-body gravitational waveforms for coalescing compact binaries with nonprecessing spins, tides and self-spin effects. Phys. Rev. D 2018, 98, 104052. [CrossRef]

49. Ossokine, S.; Buonanno, A.; Marsat, S.; Cotesta, R.; Babak, S.; Dietrich, T.; Haas, R.; Hinder, I.; Pfeiffer, H.P.; Purrer, M.; et al. Multipolar Effective-One-Body Waveforms for Precessing Binary Black Holes: Construction and Validation. Phys. Rev. D 2020, 102, 044055. [CrossRef]

50. Khan, S.; Ohme, F.; Chatziioannou, K.; Hannam, M. Including higher order multipoles in gravitational-wave models for precessing binary black holes. Phys. Rev. D 2020, 101, 024056. [CrossRef]

51. Pratten, G.; Husa, S.; Garcia-Quiros, C.; Colleoni, M.; Ramos-Buades, A.; Estelles, H.; Jaume, R. Setting the cornerstone for a family of models for gravitational waves from compact binaries: The dominant harmonic for nonprecessing quasicircular black holes. Phys. Rev. D 2020, 102, 064001. [CrossRef]

52. Berti, E.; Buonanno, A.; Will, C.M. Estimating spinning binary parameters and testing alternative theories of gravity with LISA. Phys. Rev. D 2005, 71, 084025. [CrossRef]

53. Dalal, N.; Holz, D.E.; Hughes, S.A.; Jain, B. Short grb and binary black hole standard sirens as a probe of dark energy. Phys. Rev. D 2006, 74, 063006. [CrossRef] 
54. Mishra, C.K.; Kela, A.; Arun, K.G.; Faye, G. Ready-to-use post-Newtonian gravitational waveforms for binary black holes with nonprecessing spins: An update. Phys. Rev. D 2016, 93, 084054. [CrossRef]

55. Arun, K.G.; Iyer, B.R.; Sathyaprakash, B.S.; Sundararajan, P.A. Parameter estimation of inspiralling compact binaries using 3.5 post-Newtonian gravitational wave phasing: The Non-spinning case. Phys. Rev. D 2005, 71, 084008; Erratum in 2005, $72,069903$. [CrossRef]

56. Blanchet, L. Gravitational Radiation from Post-Newtonian Sources and Inspiralling Compact Binaries. Living Rev. Rel. 2014, 17, 2. [CrossRef] [PubMed]

57. Bohé, A.; Marsat, S.; Blanchet, L. Next-to-next-to-leading order spin-orbit effects in the gravitational wave flux and orbital phasing of compact binaries. Class. Quant. Grav. 2013, 30, 135009. [CrossRef]

58. Bohé, A.; Faye, G.; Marsat, S.; Porter, E.K. Quadratic-in-spin effects in the orbital dynamics and gravitational-wave energy flux of compact binaries at the 3PN order. Class. Quant. Grav. 2015, 32, 195010. [CrossRef]

59. Marsat, S. Cubic order spin effects in the dynamics and gravitational wave energy flux of compact object binaries. Class. Quant. Grav. 2015, 32, 085008. [CrossRef]

60. Chatziioannou, K.; Poisson, E.; Yunes, N. Improved next-to-leading order tidal heating and torquing of a Kerr black hole. Phys. Rev. D 2016, 94, 084043. [CrossRef]

61. Isoyama, S.; Nakano, H. Post-Newtonian templates for binary black-hole inspirals: The effect of the horizon fluxes and the secular change in the black-hole masses and spins. Class. Quant. Grav. 2018, 35, 024001. [CrossRef]

62. Hughes, S.A. Bound orbits of a slowly evolving black hole. Phys. Rev. D 2019, 100, 064001. [CrossRef]

63. Nakano, H.; Takahashi, H.; Tagoshi, H.; Sasaki, M. An Effective search method for gravitational ringing of black holes. Phys. Rev. D 2003, 68, 102003. [CrossRef]

64. Nakano, H.; Takahashi, H.; Tagoshi, H.; Sasaki, M. An Improved search method for gravitational ringing of black holes. Prog. Theor. Phys. 2004, 111, 781-805. [CrossRef]

65. Berti, E.; Cardoso, V.; Will, C.M. On gravitational-wave spectroscopy of massive black holes with the space interferometer LISA. Phys. Rev. D 2006, 73, 064030. [CrossRef]

66. Nakano, H.; Tanaka, T.; Nakamura, T. Possible golden events for ringdown gravitational waves. Phys. Rev. D 2015, 92, 064003. [CrossRef]

67. Mohanty, S.D. Hierarchical search strategy for the detection of gravitational waves from coalescing binaries: Extension to postNewtonian wave forms. Phys. Rev. D 1998, 57, 630-658. [CrossRef]

68. Healy, J.; Lousto, C.O.; Zlochower, Y. Remnant mass, spin, and recoil from spin aligned black-hole binaries. Phys. Rev. D 2014, 90, 104004. [CrossRef]

69. Hofmann, F.; Barausse, E.; Rezzolla, L. The final spin from binary black holes in quasi-circular orbits. Astrophys. J. Lett. 2016, 825, L19. [CrossRef]

70. Healy, J.; Lousto, C.O. Remnant of binary black-hole mergers: New simulations and peak luminosity studies. Phys. Rev. D 2017, 95, 024037. [CrossRef]

71. Jiménez-Forteza, X.; Keitel, D.; Husa, S.; Hannam, M.; Khan, S.; Pürrer, M. Hierarchical data-driven approach to fitting numerical relativity data for nonprecessing binary black holes with an application to final spin and radiated energy. Phys. Rev. D 2017, 95, 064024. [CrossRef]

72. Healy, J.; Lousto, C.O. Hangup effect in unequal mass binary black hole mergers and further studies of their gravitational radiation and remnant properties. Phys. Rev. D 2018, 97, 084002. [CrossRef]

73. Varma, V.; Gerosa, D.; Stein, L.C.; Hébert, F.; Zhang, H. High-accuracy mass, spin, and recoil predictions of generic black-hole merger remnants. Phys. Rev. Lett. 2019, 122, 011101. [CrossRef]

74. Ashtekar, A.; Krishnan, B. Isolated and dynamical horizons and their applications. Living Rev. Rel. 2004, 7, 10. [CrossRef]

75. Black Hole Perturbation Club (BHPC). Available online: https:/ / sites.google.com/view/bhpc1996/home (accessed on 2 March 2021).

76. Berti, E.; Cardoso, V.; Starinets, A.O. Quasinormal modes of black holes and black branes. Class. Quant. Grav. 2009, $26,163001$. [CrossRef]

77. Emanuele Berti's Ringdown. Available online: https://pages.jh.edu/eberti2/ringdown/ (accessed on 2 March 2021).

78. Cook, G.B.; Zalutskiy, M. Gravitational perturbations of the Kerr geometry: High-accuracy study. Phys. Rev. D 2014, 90, 124021. [CrossRef]

79. Black Hole Perturbation ToolKit. Available online: https://bhptoolkit.org/ (accessed on 2 March 2021).

80. Ajith, P.; Hannam, M.; Husa, S.; Chen, Y.; Bruegmann, B.; Dorband, N.; Muller, D.; Ohme, F.; Pollney, D.; Reisswig, C.; et al. Inspiral-merger-ringdown waveforms for black-hole binaries with non-precessing spins. Phys. Rev. Lett. 2011, 106, 241101. [CrossRef] [PubMed]

81. Moore, C.J.; Cole, R.H.; Berry, C.P.L. Gravitational-wave sensitivity curves. Class. Quant. Grav. 2015, 32, 015014. [CrossRef]

82. Finn, L.S. Detection, measurement and gravitational radiation. Phys. Rev. D 1992, 46, 5236. [CrossRef]

83. Vallisneri, M. Use and abuse of the Fisher information matrix in the assessment of gravitational-wave parameter-estimation prospects. Phys. Rev. D 2008, 77, 042001. [CrossRef]

84. Babak, S.; Gair, J.; Sesana, A.; Barausse, E.; Sopuerta, C.F.; Berry, C.P.L.; Berti, E.; Amaro-Seoane, P.; Petiteau, A.; Klein, A. Science with the space-based interferometer LISA. V: Extreme mass-ratio inspirals. Phys. Rev. D 2017, 95, 103012. [CrossRef] 
85. Giesler, M.; Isi, M.; Scheel, M.A.; Teukolsky, S. Black Hole Ringdown: The Importance of Overtones. Phys. Rev. X 2019, 9, 041060. [CrossRef]

86. Poisson, E.; Will, C.M. Gravitational waves from inspiraling compact binaries: Parameter estimation using second postNewtonian wave forms. Phys. Rev. D 1995, 52, 848-855. [CrossRef]

87. Moore, C.J.; Gerosa, D.; Klein, A. Are stellar-mass black-hole binaries too quiet for LISA? Mon. Not. R. Astron. Soc. 2019, 488, L94-L98. [CrossRef]

88. Abbott, B.P.; Abbott, R.; Abbott, T.D.; Abernathy, M.R.; Acernese, F.; Ackley, K.; Adams, C.; Adams, T.; Addesso, P.; Adhikari, R.X.; et al. Tests of general relativity with GW150914. Phys. Rev. Lett. 2016, 116, 221101; Erratum in 2018, $121,129902$. [CrossRef] [PubMed]

89. Abbott, B.P.; Abbott, R.; Abbott, T.D.; Abernathy, M.R.; Acernese, F.; Ackley, K.; Adams, C.; Adams, T.; Addesso, P.; Adhikari, R.X.; et al. Tests of General Relativity with the Binary Black Hole Signals from the LIGO-Virgo Catalog GWTC-1. Phys. Rev. D 2019, 100, 104036. [CrossRef]

90. Carson, Z.; Yagi, K. Parameterized and Consistency Tests of Gravity with GravitationalWaves: Current and Future. Proceedings 2019, 17, 5. [CrossRef]

91. Abbott, R.; Abbott, T.D.; Abraham, S.; Acernese, F.; Ackley, K.; Adams, A.; Adams, C.; Adhikari, R.X.; Adya, V.B.; Affeldt, C.; et al. Tests of General Relativity with Binary Black Holes from the second LIGO-Virgo Gravitational-Wave Transient Catalog. arXiv 2020, arXiv:2010.14529.

92. Nakano, H.; Nakamura, T.; Tanaka, T. The detection of quasinormal mode with $a / M \sim 0.95$ would prove a sphere $99 \%$ soaking in the ergoregion of the Kerr space-time. Prog. Theor. Exp. Phys. 2016, 31E02. [CrossRef]

93. Pretorius, F. Evolution of binary black hole spacetimes. Phys. Rev. Lett. 2005, 95, 121101. [CrossRef]

94. Campanelli, M.; Lousto, C.O.; Marronetti, P.; Zlochower, Y. Accurate evolutions of orbiting black-hole binaries without excision. Phys. Rev. Lett. 2006, 96, 111101. [CrossRef] [PubMed]

95. Baker, J.G.; Centrella, J.; Choi, D.I.; Koppitz, M.; van Meter, J. Gravitational wave extraction from an inspiraling configuration of merging black holes. Phys. Rev. Lett. 2006, 96, 111102. [CrossRef] [PubMed]

96. Jani, K.; Healy, J.; Clark, J.A.; London, L.; Laguna, P.; Shoemaker, D. Georgia Tech Catalog of Gravitational Waveforms. Class. Quant. Grav. 2016, 33, 204001, doi:10.1088/0264-9381/33/20/204001. [CrossRef]

97. Healy, J.; Lousto, C.O.; Zlochower, Y.; Campanelli, M. The RIT binary black hole simulations catalog. Class. Quant. Grav. 2017, 34, 224001. [CrossRef]

98. Healy, J.; Lousto, C.O.; Lange, J.; O’Shaughnessy, R.; Zlochower, Y.; Campanelli, M. Second RIT binary black hole simulations catalog and its application to gravitational waves parameter estimation. Phys. Rev. D 2019, 100, 024021. [CrossRef]

99. Boyle, M.; Hemberger, D.; Iozzo, D.A.B.; Lovelace, G.; Ossokine, S.; Pfeiffer, H.P.; Scheel, M.A.; Stein, L.C.; Woodford, C.J.; Zimmerman, A.B.; et al. The SXS Collaboration catalog of binary black hole simulations. Class. Quant. Grav. 2019, $36,195006$. [CrossRef]

100. Healy, J.; Lousto, C.O. Third RIT binary black hole simulations catalog. Phys. Rev. D 2020, 102, 104018. [CrossRef]

101. Ghosh, A.; Ghosh, A.; Johnson-McDaniel, N.K.; Mishra, C.K.; Ajith, P.; Pozzo, W.D.; Nichols, D.A.; Chen, Y.; Nielsen, A.B.; Berry, C.P.L.; et al. Testing general relativity using golden black-hole binaries. Phys. Rev. D 2016, 94, 021101. [CrossRef]

102. Ghosh, A.; Johnson-Mcdaniel, N.K.; Ghosh, A.; Mishra, C.K.; Ajith, P.; Pozzo, W.D.; Berry, C.P.L.; Nielsen, A.B.; London, L. Testing general relativity using gravitational wave signals from the inspiral, merger and ringdown of binary black holes. Class. Quant. Grav. 2018, 35, 014002. [CrossRef]

103. Hughes, S.A.; Menou, K. Golden binaries for LISA: Robust probes of strong-field gravity. Astrophys. J. 2005, 623, 689-699. [CrossRef]

104. Luna, M.; Sintes, A.M. Parameter estimation of compact binaries using the inspiral and ringdown waveforms. Class. Quant. Grav. 2006, 23, 3763-3782. [CrossRef]

105. Veitch, J.; Raymond, V.; Farr, B.; Farr, W.; Graff, P.; Vitale, S.; Aylott, B.; Blackburn, K.; Christensen, N.; Coughlin, M.; et al. Parameter estimation for compact binaries with ground-based gravitational-wave observations using the LALInference software library. Phys. Rev. D 2015, 91, 042003. [CrossRef]

106. LIGO Scientific Collaboration. LIGO Algorithm Library_LALSuite. 2018. Available online: https://lscsoft.docs.ligo.org/ lalsuite/lalsimulation/index.html (accessed on 2 March 2021).

107. Abbott, B.P.; Abbott, R.; Abbott, T.D.; Abernathy, M.R.; Acernese, F.; Ackley, K.; Adams, C.; Adams, T.; Addesso, P.; Adhikari, R.X.; et al. Properties of the Binary Black Hole Merger GW150914. Phys. Rev. Lett. 2016, 116, 241102. [CrossRef] [PubMed]

108. Abbott, T.D.; Abbott, R.; Abbott, T.D.; Abernathy, M.R.; Acernese, F.; Ackley, K.; Adams, C.; Adams, T.; Addesso, P.; Adhikari, R.X.; et al. Improved analysis of GW150914 using a fully spin-precessing waveform Model. Phys. Rev. X 2016, 6, 041014. [CrossRef]

109. Yang, H.; Yagi, K.; Blackman, J.; Lehner, L.; Paschalidis, V.; Pretorius, F.; Yunes, N. Black hole spectroscopy with coherent mode stacking. Phys. Rev. Lett. 2017, 118, 161101. [CrossRef]

110. Isoyama, S.; Sturani, R.; Nakano, H. Post-Newtonian templates for gravitational waves from compact binary inspirals. arXiv 2012, arXiv:2012.01350.

111. Biwer, C.M.; Capano, C.D.; De, S.; Cabero, M.; Brown, D.A.; Nitz, A.H.; Raymond, V. PyCBC Inference: A Python-based parameter estimation toolkit for compact binary coalescence signals. Publ. Astron. Soc. Pac. 2019, 131, 024503. [CrossRef] 
112. Ashton, G.; Hübner, M.; Lasky, P.D.; Talbot, C.; Ackley, K.; Biscoveanu, S.; Chu, Q.; Divakarla, A.; Easter, P.J.; Goncharov, B.; et al. BILBY: A user-friendly Bayesian inference library for gravitational-wave astronomy. Astrophys. J. Suppl. 2019, 241, 27. [CrossRef]

113. Ireland, B.; Birnholtz, O.; Nakano, H.; West, E.; Campanelli, M. Eccentric Binary Black Holes with Spin via the Direct Integration of the Post-Newtonian Equations of Motion. Phys. Rev. D 2019, 100, 024015. [CrossRef]

114. Abbott, R.; Abbott, T.D.; Abraham, S.; Acernese, F.; Ackley, K.; Adams, C.; Adhikari, R.X.; Adya, V.B.; Affeldt, C.; Agathos, M.; et al. GW190814: Gravitational Waves from the Coalescence of a 23 Solar Mass Black Hole with a 2.6 Solar Mass Compact Object. Astrophys. J. Lett. 2020, 896, L44. [CrossRef]

115. London, L.; Khan, S.; Fauchon-Jones, E.; García, C.; Hannam, M.; Husa, S.; Jiménez-Forteza, X.; Kalaghatgi, C.; Ohme, F.; Pannarale, F. First higher-multipole model of gravitational waves from spinning and coalescing black-hole binaries. Phys. Rev. Lett. 2018, 120, 161102. [CrossRef]

116. Sakai, K.; Oohara, K.I.; Nakano, H.; Kaneyama, M.; Takahashi, H. Estimation of starting times of quasinormal modes in ringdown gravitational waves with the Hilbert-Huang transform. Phys. Rev. D 2017, 96, 044047. [CrossRef]

117. Carullo, G.; Schaaf, L.V.D.; London, L.; Pang, P.T.H.; Tsang, K.W.; Hannuksela, O.A.; Meidam, J.; Agathos, M.; Samajdar, A.; Ghosh, A.; et al. Empirical tests of the black hole no-hair conjecture using gravitational-wave observations. Phys. Rev. D 2018, 98, 104020. [CrossRef]

118. Isi, M.; Giesler, M.; Farr, W.M.; Scheel, M.A.; Teukolsky, S.A. Testing the no-hair theorem with GW150914. Phys. Rev. Lett. 2019, 123, 111102. [CrossRef]

119. Bhagwat, S.; Forteza, X.J.; Pani, P.; Ferrari, V. Ringdown overtones, black hole spectroscopy, and no-hair theorem tests. Phys. Rev. D 2020, 101, 044033. [CrossRef]

120. Ota, I.; Chirenti, C. Overtones or higher harmonics? Prospects for testing the no-hair theorem with gravitational wave detections. Phys. Rev. D 2020, 101, 104005. [CrossRef]

121. Nakano, H.; Narikawa, T.; Oohara, K.; Sakai, K.; Shinkai, H.; Takahashi, H.; Tanaka, T.; Uchikata, N.; Yamamoto, S.; Yamamoto, T.S. Comparison of various methods to extract ringdown frequency from gravitational wave data. Phys. Rev. D 2019, $99,124032$. [CrossRef]

122. Yamamoto, T.S.; Tanaka, T. Use of conditional variational auto encoder to analyze ringdown gravitational waves. arXiv 2012, arXiv:2002.12095.

123. Carson, Z.; Yagi, K. Testing General Relativity with Gravitational Waves. arXiv 2011, arXiv:2011.02938.

124. Detweiler, S.L. Black holes and gravitational waves. III. the resonant frequencies of rotating holes. Astrophys. J. 1980, $239,292-295$. [CrossRef]

125. Dreyer, O.; Kelly, B.J.; Krishnan, B.; Finn, L.S.; Garrison, D.; Lopez-Aleman, R. Black hole spectroscopy: Testing general relativity through gravitational wave observations. Class. Quant. Grav. 2004, 21, 787-804. [CrossRef]

126. Berti, E.; Sesana, A.; Barausse, E.; Cardoso, V.; Belczynski, K. Spectroscopy of Kerr black holes with Earth- and space-based interferometers. Phys. Rev. Lett. 2016, 117, 101102. [CrossRef]

127. Baibhav, V.; Berti, E. Multimode black hole spectroscopy. Phys. Rev. D 2019, 99, 024005. [CrossRef]

128. Gnocchi, G.; Maselli, A.; Abdelsalhin, T.; Giacobbo, N.; Mapelli, M. Bounding alternative theories of gravity with multiband GW observations. Phys. Rev. D 2019, 100, 064024. [CrossRef]

129. Berti, E.; Cardoso, J.; Cardoso, V.; Cavaglia, M. Matched-filtering and parameter estimation of ringdown waveforms. Phys. Rev. D 2007, 76, 104044. [CrossRef]

130. Akutsu, T.; Ando, M.; Arai, K.; Arai, Y.; Araki, S.; Araya, A.; Aritomi, N.; Asada, H.; Aso, Y.; Bae, S.; et al. Overview of KAGRA: KAGRA science. arXiv 2020, arXiv:2008.02921.

131. Uchikata, N.; Narikawa, T.; Sakai, K.; Takahashi, H.; Nakano, H. Black hole spectroscopy for KAGRA future prospect in O5. Phys. Rev. D 2020, 102, 024007. [CrossRef]

132. Tso, R.; Gerosa, D.; Chen, Y. Optimizing LIGO with LISA forewarnings to improve black-hole spectroscopy. Phys. Rev. D 2019, 99, 124043. [CrossRef]

133. Lousto, C.O. (Rochester Institute of Technology, Rochester, NY, USA). Personal communication, 2020.

134. Romero-Shaw, I.M.; Lasky, P.D.; Thrane, E.; Bustillo, J.C. GW190521: Orbital eccentricity and signatures of dynamical formation in a binary black hole merger signal. Astrophys. J. Lett. 2020, 903, L5. [CrossRef]

135. Bustillo, J.C.; Sanchis-Gual, N.; Torres-Forné, A.; Font, J.A. Confusing head-on and precessing intermediate-mass binary black hole mergers. arXiv 2020, arXiv:2009.01066.

136. Peters, P.C.; Mathews, J. Gravitational radiation from point masses in a Keplerian orbit. Phys. Rev. 1963, 131, 435-439. [CrossRef]

137. Peters, P.C. Gravitational Radiation and the Motion of Two Point Masses. Phys. Rev. 1964, 136, B1224. [CrossRef]

138. Maggiore, M. Gravitational Waves. Vol. 1: Theory and Experiments; Oxford University Press: Oxford, UK, 2014.

139. Amaro-Seoane, P. Detecting Intermediate-Mass Ratio Inspirals From The Ground And Space. Phys. Rev. D 2018, 98, 063018. [CrossRef]

140. Holgado, A.M.; Ortega, A.; Rodriguez, C.L. Dynamical Formation Scenarios for GW190521 and Prospects for Decihertz Gravitational-Wave Astronomy with GW190521-Like Binaries. arXiv 2020, arXiv:2012.09169.

141. Amaro-Seoane, P.; Miller, M.C.; Freitag, M. Gravitational waves from eccentric intermediate-mass black hole binaries. Astrophys. J. Lett. 2009, 692, L50-L53. [CrossRef]

142. Loutrel, N. Repeated Bursts: Gravitational Waves from Highly Eccentric Binaries. arXiv 2020, arXiv:2009.11332. 
143. Nagar, A.; Bonino, A.; Rettegno, P. All in one: Effective one body multipolar waveform model for spin-aligned, quasi-circular, eccentric, hyperbolic black hole binaries. arXiv 2021, arXiv:2101.08624.

144. Arredondo, J.N.; Loutrel, N. Neutron Stars in the Effective Fly-By Framework: f-Mode Re-summation. arXiv 2021, arXiv:2101.10963.

145. Setyawati, Y.; Ohme, F. Adding eccentricity to quasi-circular binary-black-hole waveform models. arXiv 2021, arXiv:2101.11033.

146. Islam, T.; Varma, V.; Lodman, J.; Field, S.E.; Khanna, G.; Scheel, M.A.; Pfeiffer, H.P.; Gerosa, D.; Kidder, L.E. Eccentric binary black hole surrogate models for the gravitational waveform and remnant properties: Comparable mass, nonspinning case. arXiv 2021, arXiv:2101.11798.

147. Finn, L.S.; Thorne, K.S. Gravitational waves from a compact star in a circular, inspiral orbit. Phys. Rev. D 2000, 62, 124021. [CrossRef]

148. Huerta, E.A.; McWilliams, S.T.; Gair, J.R.; Taylor, S.R. Detection of eccentric supermassive black hole binaries with pulsar timing arrays: Signal-to-noise ratio calculations. Phys. Rev. D 2015, 92, 063010. [CrossRef]

149. Cutler, C.; Kennefick, D.; Poisson, E. Gravitational radiation reaction for bound motion around a Schwarzschild black hole. Phys. Rev. D 1994, 50, 3816-3835. [CrossRef]

150. Gondán, L.; Kocsis, B.; Raffai, P.; Frei, Z. Accuracy of Estimating Highly Eccentric Binary Black Hole Parameters with GravitationalWave Detections. Astrophys. J. 2018, 855, 34. [CrossRef]

151. Mikoczi, B.; Kocsis, B.; Forgacs, P.; Vasuth, M. Parameter estimation for inspiraling eccentric compact binaries including pericenter precession. Phys. Rev. D 2012, 86, 104027. [CrossRef]

152. Drasco, S.; Hughes, S.A. Gravitational wave snapshots of generic extreme mass ratio inspirals. Phys. Rev. D 2006, 73, 024027; Erratum in 2013, 88, 109905; Erratum in 2014, 90, 109905. [CrossRef]

153. Nishizawa, A.; Berti, E.; Klein, A.; Sesana, A. eLISA eccentricity measurements as tracers of binary black hole formation. Phys. Rev. D 2016, 94, 064020. [CrossRef]

154. Seto, N. Prospects of eLISA for Detecting Galactic Binary Black Holes Similar to GW150914. Mon. Not. R. Astron. Soc. 2016, 460, L1-L4. [CrossRef]

155. Abbott, R.; Abbott, T.D.; Abraham, S.; Acernese, F.; Ackley, K.; Adams, A.; Adams, C.; Adhikari, R.X.; Adya, V.B.; Affeldt, C.; et al. Population Properties of Compact Objects from the Second LIGO-Virgo Gravitational-Wave Transient Catalog. arXiv 2020, arXiv:2010.14533.

156. Kinugawa, T.; Nakamura, T.; Nakano, H. Formation of mass gap compact object and black hole binary from Population III stars. arXiv 2007, arXiv:2007.13343.

157. KAGRA Collaboration; LIGO Scientific Collaboration; Virgo Collaboration. Prospects for Observing and Localizing GravitationalWave Transients with Advanced LIGO, Advanced Virgo and KAGRA. Living Rev. Relativ. 2020, 21, 3. [CrossRef]

158. Hild, S.; Chelkowski, S.; Freise, A. Pushing towards the ET sensitivity using 'conventional' technology. arXiv 2008, arXiv:0810.0604.

159. Mishra, C.K.; Arun, K.G.; Iyer, B.R.; Sathyaprakash, B.S. Parametrized tests of post-Newtonian theory using Advanced LIGO and Einstein Telescope. Phys. Rev. D 2010, 82, 064010. [CrossRef]

160. ET Sensitivities Page. Available online: http:/ /www.et-gw.eu/index.php/etsensitivities (accessed on 2 March 2021).

161. Yagi, K.; Seto, N. Detector configuration of DECIGO/BBO and identification of cosmological neutron-star binaries. Phys. Rev. D 2011, 83, 044011; Erratum in 2017, 95, 109901. [CrossRef]

162. Hu, X.C.; Li, X.H.; Wang, Y.; Feng, W.F.; Zhou, M.Y.; Hu, Y.M.; Hu, S.C.; Mei, J.W.; Shao, C.G. Fundamentals of the orbit and response for TianQin. Class. Quant. Grav. 2018, 35, 095008. [CrossRef]

163. Shi, C.; Bao, J.; Wang, H.; Zhang, J.D.; Hu, Y.; Sesana, A.; Barausse, E.; Mei, J.; Luo, J. Science with the TianQin observatory: Preliminary results on testing the no-hair theorem with ringdown signals. Phys. Rev. D 2019, 100, 044036. [CrossRef] 